\title{
Backward Stochastic Differential Equations with Nonmarkovian Singular Terminal Values
}

\author{
Ali Devin Sezer *, Thomas Kruse ${ }^{\dagger}$, Alexandre Popier ${ }^{\ddagger}$
}

January 15, 2018

\begin{abstract}
We solve a class of BSDE with a power function $f(y)=y^{q}, q>1$, driving its drift and with the terminal boundary condition $\xi=\infty \cdot \mathbf{1}_{B(m, r)^{c}}$ (for which $q>2$ is assumed) or $\xi=\infty \cdot \mathbf{1}_{B(m, r)}$, where $B(m, r)$ is the ball in the path space $C([0, T])$ of the underlying Brownian motion centered at the constant function $m$ and radius $r$. The solution involves the derivation and solution of a related heat equation in which $f$ serves as a reaction term and which is accompanied by singular and discontinuous Dirichlet boundary conditions. Although the solution of the heat equation is discontinuous at the corners of the domain the BSDE has continuous sample paths with the prescribed terminal value.
\end{abstract}

AMS 2010 class: 35K57, 35K67, 60G40, 60H30, 60H99, 60J65

Keywords: Backward stochastic differential equations / Reaction-diffusion equations / Singularity / Non-Markovian terminal conditions

\section{Introduction}

One of the first points emphasized in an introductory ordinary differential equations (ODE) course is that the solution of an ODE may explode in finite time; the equation

$$
\frac{d y}{d t}=y^{q}
$$

with $q>1$ serves as the primary example. Indeed, specify the terminal value $y_{T}=\infty$ to (1) and

$$
y_{t} \doteq((q-1)(T-t))^{1-p}, \quad t<T, \quad 1 / p+1 / q=1,
$$

will be the corresponding unique solution of (1) ( $p$ is the Hölder conjugate of $q$ ). Now let $W$ be a standard Brownian motion and $\left\{\mathscr{F}_{t}\right\}$ be its natural filtration. For a terminal condition $\xi \in \mathscr{F}_{T}$, one can think of the backward stochastic differential equation (BSDE)

$$
\begin{aligned}
Y_{s} & =Y_{t}+\int_{s}^{t} f\left(Y_{r}\right) d r+\int_{s}^{t} Z_{r} d W_{r}, \quad 0<s<t<T, \\
Y_{T} & =\xi
\end{aligned}
$$

*Middle East Technical University, Institute of Applied Mathematics, Ankara, Turkey, devin@metu.edu.tr

${ }^{\dagger}$ University of Duisburg-Essen, Thea-Leymann-Str. 9, 45127 Essen, Germany, e-mail: thomas.kruse@uni-due.de

${ }^{\ddagger}$ Laboratoire Manceau de Mathématiques, Université du Maine, Avenue Olivier Messiaen, 72085 Le Mans, Cedex 9, France. e-mail: alexandre.popier@univ-lemans.fr

We are grateful to an anonymous referee for helpful comments and suggestions. 
$f(y)=-y|y|^{q-1}, Y$ continuous $^{1}$ on $[0, T]$, as a stochastic generalization / perturbation of the ODE(1) because for $\xi=\infty$ identically, one can set $Z_{t}=0$ and reduce (3) to (1) for which $Y_{t}=y_{t}$ is the unique solution. But $\xi$ is a random variable and can also be chosen equal $\infty$ over a measurable set $A \in \mathscr{F}_{T}$ and a finite random variable over $A^{c}$. Can one solve the BSDE $(3,4)$ with such terminal conditions? An analysis of this and related questions began with the article [23], where $W$ is assumed to be $d$-dimensional. [23] proved in particular that there exists a pair of processes $\left(Y^{\mathrm{min}}, Z^{\mathrm{min}}\right)$ adapted to the filtration $\mathscr{F}_{t}$ satisfying (3) and where $Y^{\mathrm{min}}$ satisfies almost surely (a.s.)

$$
\lim _{t \rightarrow T} Y_{t}^{\min } \geq \xi=Y_{T}^{\min } .
$$

In other words, the process $Y^{\text {min }}$ is a continuous process on $[0, T)$, whose left-limit as $t$ goes to $T$ exists a.s. and dominates the terminal condition $\xi=Y_{T}^{\min }$. Moreover, $Y^{\min }$ of [23] is minimal: for any other pair $(\hat{Y}, \hat{Z})$ satisfying $(3)$ and

$$
\liminf _{t \rightarrow T} \hat{Y}_{t} \geq \xi
$$

one has

$$
Y_{t}^{\min } \leq \hat{Y}_{t} \text {, a.s., } t \in[0, T] \text {. }
$$

Following [16] we will refer to any pair satisfying (3) and (6) as a super-solution of the BSDE $(3,4)$. Thus $\left(Y^{\mathrm{min}}, Z^{\mathrm{min}}\right)$ is the minimal super-solution of the BSDE $(3,4)$. To strengthen (5) to the a.s. equality

$$
\lim _{t \rightarrow T} Y_{t}^{\min }=Y_{T}^{\min }=\xi
$$

and hence solving the $\operatorname{BSDE}(3,4)$ for general $\xi \in \mathscr{F}_{T}$ turns out to be a difficult problem. The article [23] proved (8) for $\xi$ of the form $\xi=g\left(W_{T}\right)$, where the function $g: \mathbb{R} \mapsto \mathbb{R}_{+} \cup\{\infty\}$ satisfies $\{g=\infty\}$ is closed and for any compact subset $K$ of $\{g<\infty\}, \mathbb{E}\left[g\left(W_{T}\right) \mathbf{1}_{K}\left(W_{T}\right)\right]<$ $\infty$. Because $\xi$ is a deterministic function of $W$, such terminal conditions are referred to as "Markovian". To the best of our knowledge, to delineate the class of $\xi \in \mathscr{F}_{T}$ for which the BSDE $(3,4)$ has a solution $Y$ on $[0, T]$ still remains an open problem.

The goal of the present work is to construct solutions to the BSDE $(3,4)$ for a class of nonMarkovian terminal conditions $\xi \in \mathscr{F}_{T}$; we will also prove that the solutions we construct are equal to the minimal supersolutions $\left(Y^{\mathrm{min}}, Z^{\mathrm{min}}\right)$ of [23], which will imply that (8) holds for the terminal conditions we treat. To the best of our knowledge, the present work is the first to derive these types of results for non-Markovian singular terminal conditions. The class of $\xi$ which we will focus on is best explained using the canonical path space $\Omega \doteq C([0, T], \mathbb{R})$, the set of all $\mathbb{R}$-valued continuous paths $\omega$ on $[0, T]$, equipped with its sup norm

$$
\|\omega\|_{\infty} \doteq \sup _{t \in[0, T]}|\omega(t)|
$$

and a family of Wiener measures $\left\{\mathbb{P}^{x}, x \in \mathbb{R}\right\}$, under which the canonical process $W_{t}(\omega)=$ $\omega(t)$ is a standard Brownian motion with initial condition $W_{0}=x$. As before $\left\{\mathscr{F}_{t}, 0 \leq t \leq T\right\}$ is the canonical filtration generated by $W$. Then $\mathscr{F}_{T}$ is the Borel field of $\Omega$ corresponding to the sup norm $\|\cdot\|_{\infty}$ and the basic $\mathscr{F}_{T}$-measurable random variables are the indicator

\footnotetext{
${ }^{1}$ The requirement that $Y$ be continuous on $[0, T]$ is natural- otherwise one could solve the SDE (3) arbitrarily on $[0, T)$ and set $Y_{T}=\xi$; thus the terminal condition $\xi$ would have no bearing on the behavior of $(Y, Z)$ on $[0, T)$.
} 
functions of open / closed subsets of $C([0, T])$. The open and closed subset of $C([0, T])$ are generated by balls with respect to the norm $\|\cdot\|_{\infty}$ and the simplest balls in turn are those centered around constant functions. Thus we arrive at the class of terminal values we would like to cover:

$$
\xi_{1}=\infty \cdot \mathbf{1}_{B(m, r)^{c}} \quad \text { or } \quad \xi_{2}=\infty \cdot \mathbf{1}_{B(m, r)}
$$

where $B(m, r)$ is the ball $\left\{\omega:\|\omega-m\|_{\infty} \leq r\right\}$, for some $m \in \mathbb{R}$ and $r>0$. To simplify notation we will assume throughout that $m=r=L / 2$ for some $L>0$ for which the expressions for $\xi$ in (9) become $\xi_{1}=\infty \cdot \mathbf{1}_{B(L / 2, L / 2)^{c}}$ and $\xi_{2}=\infty \cdot \mathbf{1}_{B(L / 2, L / 2)}$; all of what follows trivially extends to arbitrary $m \in \mathbb{R}$ and $r>0$.

The Markovian terminal conditions provide (via Itô's formula) the connection between BSDE and a class of semilinear / quasilinear parabolic PDE [22]. In the case of singular terminal conditions of the type $g\left(X_{T}\right)$ where $g$ can take the value $+\infty$, the associated parabolic PDE is coupled with singular boundary conditions; a considerable number of articles appeared over the last several decades (see $[2,3,19,6,18]$ and the references therein) studying the PDE

$$
\partial_{t} V+\frac{1}{2} \partial_{x x} V-V^{q}=0
$$

allowing for singular terminal values. This PDE (10) is directly related to the BSDE $(3,4)$ and will play a key role in our analysis below. See [23, Section 4] for more on the link between the BSDE $(3,4)$ and the PDE (10).

The main idea of the present paper for the solution of the BSDE $(3,4)$ for $\xi$ of the form (9) is to reduce the question to a Markovian problem in the random time interval $[0, \tau \wedge T]$ where

$$
\tau \doteq \inf \left\{t \in[0, \infty): W_{t} \in\{0, L\}\right\}, \quad W_{0}=x, x \in(0, L) .
$$

For $\tau<T$, the terminal conditions given in (9) reduce to constants

$$
\xi_{1}(\omega)=\infty \cdot \mathbf{1}_{B(m, r)^{c}}=\infty, \quad \xi_{2}(\omega)=\infty \cdot \mathbf{1}_{B(m, r)}=0,
$$

and the SDE (3) reduces to the ODE (1) on $(\tau, T]$. Solving it on $(\tau, T]$ with the terminal condition $\xi_{1}(\omega)=\infty$ gives the solution

$$
Y_{t}^{1}=y_{t}, Z_{t}^{1}=0, \quad t \in(\tau, T],
$$

of the BSDE $(3,4)$ on $(\tau, T]$ for $\xi=\xi_{1}$. Similarly, solving the same ODE on the same time interval with the terminal condition $\xi_{2}(\omega)=0$ gives the solution

$$
Y_{t}^{2}=0, Z_{t}^{2}=0, \quad t \in(\tau, T]
$$

of the same BSDE for $\xi=\xi_{2}$. These then give the value of the solutions $Y^{i}$ at time $\tau<T$ :

$$
Y_{\tau}^{1}=y_{\tau}, Y_{\tau}^{2}=0 .
$$

On the set $T<\tau$, the terminal conditions $\xi_{1}$ and $\xi_{2}$ reduce to

$$
\xi_{1}(\omega)=0, \quad \xi_{2}(\omega)=\infty .
$$

Next we solve the same BSDE in the time interval $[0, T \wedge \tau]$ using (11) and (12) as terminal conditions. Thus our BSDE is reduced to one with a Markovian terminal condition at the 
random terminal time $\tau \wedge T$. Now Itô's formula provides the connection between the solution of the reduced BSDE to the solution of the parabolic equation (10)

$$
\partial_{t} V+\frac{1}{2} \partial_{x x} V-V^{q}=0
$$

(11) and (12) suggest the following boundary conditions to accompany the PDE:

$$
V(0, t)=V(L, t)=y_{t}, t \in[0, T], \quad V(x, T)=0,0<x<L
$$

for $\xi_{1}$ and

$$
V(0, t)=V(L, t)=0, t \in[0, T], \quad V(x, T)=\infty, 0<x<L
$$

for $\xi_{2}$; (note that in a single space dimension the Laplacian reduces to the second derivative operator $\left.\partial_{x x}\right)$. Proposition 1 of Section 2 gives the details of the above reduction.

With these steps our problem is reduced to the solution of the PDE (10) and the boundary condition (13) for $\xi_{1}$ and the boundary condition (14) for $\xi_{2}$. The main difficulty with the solution of these equations are the discontinuous (at the corners $(0, T),(L, T) \in \mathbb{R}^{2}$ ) and infinite valued boundary conditions. The most relevant work that we have identified in the literature on the solution of (10) and the boundary conditions (13) and (14) is [19], which contains results giving the existence of weak solutions to the PDE (10) in $d$ space dimensions when coupled with boundary conditions which are allowed to take the value $+\infty$. These results occur in [19] in the context of the computation of initial traces and within a general framework where boundary conditions and solutions are specified in a weak Sobolev-sense; to treat these questions the authors of [19] use PDE and analysis results developed by them over a number of works. We think that one can build an argument starting from results in [19] to get a classical solution to $(10,13)$ and $(10,14)$ having the regularity and the boundary continuity properties needed for our purposes but this appears to be a nontrivial task. In this paper, we follow a different route and give a new self contained construction of classical solutions of $(10,13)$ and $(10,14)$ starting from classical parabolic PDE theory with smooth boundary conditions [8] and building on it using smooth approximation from below of the boundary conditions and elementary probabilistic techniques.

Once the solution of the BSDE is built as above, the last step is to connect it with the corresponding minimal supersolution $\left(Y^{\mathrm{min}}, Z^{\mathrm{min}}\right)$; this is achieved by an argument using the approximating sequence of functions constructed in the solution of the PDE.

One change in the application of the above steps to the terminal conditions $\xi_{1}$ and $\xi_{2}$ is the assumption we make on $q$ : for $\xi_{1}$ we need $q>2$ whereas $q>1$ suffices for $\xi_{2}$. This is coupled with the following change in the argument: for $q>2$, the classical heat equation $\partial_{t} V+\frac{1}{2} \partial_{x x}^{2} V=0$ also has a classical solution $v_{0}$ with the boundary condition (13). In the treatment of $\xi_{1}$ we use $v_{0}$ as an upper bound in constructing an approximating sequence for the solution of (10) and (13), which ensures the continuity of the limit of the approximation at the boundaries. For $\xi_{2}$ the corresponding boundary condition is (14), for which $v_{0}$ doesn't exist (regardless of the value of $q$ ) but we are able to construct an upperbound directly working with the PDE (10) and the boundary condition (14) and for this $q>1$ suffices. Other than this, the arguments for $\xi_{1}$ and $\xi_{2}$ are the same. To reduce repetition and shorten the paper we give them in detail for the first case in Section 2, the necessary changes for $\xi_{2}$ are given in Section 3. The results of these sections are summarily given in Theorems 1 (Section 2) and 2 (Section 3). Both of these sections present numerical examples (graphs of functions and example sample paths) of the constructed solutions of the BSDE and those of the associated PDE. 
We would like to note a connection between our results and the BSDE theory with $L^{p}$ terminal conditions. The assumption $q>2$ for $\xi_{1}$ implies that, with the above reduction of the $\operatorname{BSDE}(3,4)$ to the random time interval $[0, \tau \wedge T]$, the reduced terminal condition will be in $L^{1}$; thus one can also invoke the existence results of [4] to construct a solution for the terminal condition $\xi_{1}$. The reduction to the time interval $[0, \tau \wedge T]$ doesn't lead to an $L^{1}$ terminal condition for $\xi=\xi_{2}$; the PDE approach above applies to both $\xi_{1}$ and $\xi_{2}$.

A well known fact in the prior literature (see, e.g.,[16]) is the link between the BSDE $(3,4)$ and the following stochastic optimal control problem: the controlled process $C$ is $C_{s}=c+\int_{s}^{t} \alpha_{s} d s$, the running cost is $|\alpha|^{p}$ and the terminal cost is $\left|C_{T}\right|^{p} \xi$, where $0 \cdot \infty=0$. The random variable $\xi$ is a penalty on the terminal value of $C$; in particular the controlled process is constrained to satisfy $C_{T}=0$ if $\xi=+\infty$. A growing number of articles study variants and generalizations of this control problem (with $\xi=\infty$ identically) with applications to liquidation of portfolios of assets, see $[1,13,12,16]$. The value function $v$ of the control problem is given by the minimal solution $Y^{\min }: v\left(t_{0}, c\right)=|c|^{p} Y_{t_{0}}^{\min }$, where $Y_{t_{0}}^{\min }$ is the initial value of the solution of the $\operatorname{BSDE}(3,4)$ when it is solved on the interval $\left[t_{0}, T\right]$ with $W_{t_{0}}=x$ [16]. Therefore, our results in Section 2 and 3 give explicit expressions for the value function of this control problem for $\xi=\infty \cdot \mathbf{1}_{B(m, r)}$ and $\xi=\infty \cdot \mathbf{1}_{B(m, r)^{c}}$. Section 4 uses this connection to derive estimates on the conditional probabilities $\mathbb{P}\left(B(m, r) \mid \mathscr{F}_{t}\right)$ and $\mathbb{P}\left(B(m, r)^{c} \mid \mathscr{F}_{t}\right), t \in[0, T)$.

Let us point out further prior literature on the solution of the BSDE (3,4): [23] considers the case where $\xi$ is a function $g\left(X_{T}\right)$ where $X$ is the solution of a forward SDE

$$
X_{t}=x+\int_{0}^{t} b\left(s, X_{s}\right) d r+\int_{0}^{t} \sigma\left(s, X_{s}\right) d W_{s}
$$

(for the assumptions on $b, \sigma$ and $q$ we refer the reader to [23]). Since then, two works $[21,24]$ appeared treating the BSDE $(3,4)$ both focusing on $\xi$ of the form $g\left(X_{T}\right)$. The work [21] extends the results of [23] to the class of backward doubly stochastic SDE (BDSDE in short). The article [21] proves under these models that a minimal super-solution $\left(Y^{\mathrm{min}}, Z^{\mathrm{min}}\right)$ exists which is also continuous at the terminal time $T$ with $Y_{T}^{\min }=\xi=g\left(X_{T}\right)$. The work [24] also considers the BSDE with three additional extensions a) there are an additional jump term given by a Poisson random measure; b) the drift term $f\left(Y_{s}\right)=Y_{s}^{q}$ in (3) is replaced with a general $f$ satisfying a number of conditions which includes as a special case the function $y \rightarrow y^{q}$ and c) it works with a general complete right continuous filtration to which all of the given processes are adapted (as in [16]); [24] proves that under these model assumptions that the minimal super-solution $Y^{\mathrm{min}}$ to the BSDE is continuous at the terminal time with $Y_{T}^{\min }=\xi=g\left(X_{T}\right)$ (in [24] jump terms are also allowed in the dynamics of $X$ ). Note that existence and minimality of $\left(Y^{\mathrm{min}}, Z^{\mathrm{min}}, U^{\mathrm{min}}, M^{\mathrm{min}}\right)$ were proved already in [16] (the terms $U^{\text {min }}$ and $M^{\text {min }}$ come from the Poisson measure and the general filtration). A recent work treating integro-partial differential generalizations of (10) with singular terminal conditions is [25], which contains many further references and a literature review on parabolic PDE with singular boundary conditions, their connections to BSDE and their probabilistic solutions.

We indicate several directions for future research in the Conclusion.

\section{A first non-Markovian case}

This section implements for the terminal condition $\xi=\xi_{1}=\infty \cdot \mathbf{1}_{B(L / 2, L / 2)^{c}}$ the argument whose outline was given in the introduction. We will denote by $D$ the domain $(0, L) \times$ 
$(0, T)$. For $x \notin(0, L), \mathbb{P}^{x}(\xi=+\infty)=1$ and the problem becomes trivial for such $x$ (the same comment applies to the terminal condition $\xi=\infty \cdot \mathbf{1}_{B(L / 2, L / 2)}$ with $\left.\mathbb{P}^{x}(\xi=0)=1\right)$. Therefore, will assume the initial condition $x$ to satisfy $x \in(0, L)$; none of the arguments of the present work depend on the initial point $W_{0}=x$ beyond this consideration, thus for ease of notation we will simply write $\mathbb{P}$ for $\mathbb{P}^{x}$ and always assume $x \in(0, L)$. We summarize the results of this section in the following Theorem.

Theorem 1. If $q>2$ then there is a function $u$ which is $C^{\infty}$ in the $x$ variables and $C^{1}$ in the $t$ variable and continuous on $\bar{D} \backslash\{(L, T),(0, T)\}$ satisfying the PDE (10) with the boundary condition (13) such that

1.

$$
Y_{t}=\left\{\begin{array}{ll}
u\left(W_{t}, t\right) & , t<\tau \wedge T, \\
y_{t} & , \tau \leq t \leq T,
\end{array} \quad Z_{t}= \begin{cases}u_{x}\left(W_{t}, t\right) & , t<\tau \wedge T \\
0 & , \tau \leq t \leq T\end{cases}\right.
$$

solve the $B S D E(3,4)$ with $\xi=\xi_{1}=\infty \cdot \mathbf{1}_{B(L / 2, L / 2)^{c}}$; in particular, $Y$ is continuous on $[0, T]$,

2. We have $\left(Y^{\min }, Z^{\min }\right)=(Y, Z)$; in particular (8) holds.

Proof. Proposition 1 of subsection 2.1 proves that given any classical solution $u$ of (10) and the boundary condition (13), the processes $(Y, Z)$ defined as in (15) satisfy the BSDE $(3,4)$ and the $Y$ process is continuous on $[0, T]$. Proposition 2 of subsection 2.2 constructs a classical solution $u$ of (10) and the boundary condition (13). Finally, Proposition 3 proves $Y=Y^{\mathrm{min}}$ for the $u$ constructed in Proposition 2, which implies in particular that, for $\xi=\xi_{1}$, (8) holds.

Remark 1. As pointed out in the introduction, the connection between the BSDE $(3,4)$ and the PDE (10) is well known for Markovian terminal conditions. The above result says that the same connection continues to hold when ones uses the non-Markovian $\xi_{1}$ as terminal condition for the BSDE.

We give several numerical examples and simulation of our results in subsection 2.4.

\subsection{Reduction to heat equation with reaction}

As outlined in the introduction, our approach to solving the $\operatorname{BSDE}(3,4) \xi=\infty \cdot \mathbf{1}_{B(L / 2, L / 2)^{c}}$ will be by breaking the problem into two random time intervals $[0, \tau \wedge T)$ and $(\tau \wedge T, T]$; on the latter the problem reduces to the trivial (1) with the terminal value $y_{T}=\infty$. The value of the unique solution $y_{\tau}$ at $\tau$ then provides the terminal condition over the interval $[0, \tau \wedge T)$; thus we end up with a Markovian problem and can attack it via the associated PDE. These are the main ideas underlying the next proposition.

Proposition 1. Suppose $u: \bar{D} \rightarrow \mathbb{R}$ is $C^{\infty}$ in the $x$ variable and continuously differentiable in the $t$ variable over $D$, continuous on $\bar{D} \backslash\{(L, T),(0, T)\}$ and satisfies the PDE (10) and the boundary condition (13) in the classical sense. Then the pair $(Y, Z)$ of (15) satisfies the $B S D E(3,4)$ and is continuous on $[0, T]$.

Proof. We begin by proving that $Y$ is continuous on $[0, T]$. First consider the case $\{\tau<$ $T\}$. By assumption $u$ is continuous on $\bar{D} \backslash\{(L, T),(0, T)\}$. Therefore, $u$ is continuous on $[0, L] \times[0, \tau],[0, \tau] \varsubsetneqq[0, T]$. In addition, $W$ has continuous sample paths. Then $t \mapsto u\left(W_{t}, t\right)$ 
is the composition of two continuous maps on $[0, \tau]$ and therefore is a continuous function on that interval. On the other hand, by definition (15) $Y_{t}=y_{t}$ for $t>\tau$; and the continuity of $t \mapsto y_{t}$ on $[\tau, T]$ implies the same for $Y$; finally the continuity of $Y$ at $\tau$ follows from the boundary condition (13) and the definition of $Y$ given in (15): $u\left(W_{\tau}, \tau\right)=y_{\tau}=Y_{\tau}$. Thus we see that $Y$ is continuous on $[0, T]$ on the set $\{\tau<T\}$. The event $\{\tau=T\}$ is of measure zero, thus it only remains to consider the case $\{\tau>T\}$. By definition (15) $Y_{t}=u\left(W_{t}, t\right)$, $t \in[0, T]$ for $\omega \in\{\tau>T\}$. The continuity of the sample path of $W$ and the compactness of $[0, T]$ imply that there exists $\delta>0$ such that

$$
W_{t}(\omega) \in[\delta, L-\delta], t \in[0, T],
$$

for $\omega \in\{\tau>T\}$. By assumption $u$ is continuous on $[\delta, L-\delta] \times[0, T]$. Then $t \mapsto Y_{t}=u\left(W_{t}, t\right)$, $t \in[0, T]$ is the composition of two continuous functions and hence continuous. This proves the continuity of $Y$ on $[0, T]$.

By definition

$$
\begin{aligned}
Y_{T} & =y_{T} \cdot \mathbf{1}_{\{\tau<T\}}+u\left(W_{T}, T\right) \mathbf{1}_{\{\tau>T\}} \\
& =\infty \cdot \mathbf{1}_{B(L / 2, L / 2)^{c}}+u\left(W_{T}, T\right) \mathbf{1}_{\{\tau>T\}} .
\end{aligned}
$$

The fact (16) and that $u$ satisfies (13) imply $u\left(W_{T}, T\right) \mathbf{1}_{\{\tau>T\}}=0$. This and the last display imply $Y_{T}=\xi_{1}$, i.e., that $Y$ satisfies the terminal condition (4) with $\xi=\xi_{1}$.

It remains to prove that for fixed $s<t<T$ (3) holds almost surely. On the set $\{\tau \leq s\}$, $Y_{r}=y_{r}$ and $Z_{r}=0$ for $r \in[s, t]$ and (3) reduces to

$$
y_{t}=y_{s}+\int_{s}^{t} y_{r}^{q} d r
$$

which is equivalent to (1) of which $y$ is a solution; this establishes that (3) holds over $\{\tau \leq s\}$. Recall that by assumption, $u$ is smooth in $x$, continuously differentiable in $t$ in $D$ and continuous on $\bar{D} \backslash\{(L, T),(0, T)\}$. In particular, $u$ is continuous on any $[0, L] \times[0, t]$ for $t<T$. On the set $\{\tau>s\}$ apply Itô's formula to $u\left(W_{r}, r\right)$ between $s$ and $\tau \wedge t$ to get

$$
Y_{\tau \wedge t}=Y_{s}+\int_{s}^{t \wedge \tau} \partial_{x}\left(W_{r}, r\right) d W_{r}+\int_{s}^{t \wedge \tau} \partial_{t} u\left(W_{r}, r\right) d r+\frac{1}{2} \int_{s}^{t \wedge \tau} \partial_{x x} u\left(W_{r}, r\right) d r .
$$

That $u$ satisfies (10) implies

$$
\begin{aligned}
Y_{\tau \wedge t} & =Y_{s}+\int_{s}^{t \wedge \tau} \partial_{x} u\left(W_{r}, r\right) d W_{r}+\int_{s}^{t \wedge \tau} u^{q}\left(W_{r}, r\right) d r \\
& =Y_{s}+\int_{s}^{t \wedge \tau} Z_{r} d W_{r}+\int_{s}^{t \wedge \tau} Y_{r}^{q} d r
\end{aligned}
$$

which implies (3) for $\{\tau>t\}$. Finally, for $\{\tau \in(s, t)\}$ :

$$
Y_{t}=Y_{\tau}+\int_{\tau}^{t} Y_{r}^{q} d r
$$

Substituting the right side of (17) for $Y_{\tau}$ in the last display gives

$$
Y_{t}=Y_{s}+\int_{s}^{t} Z_{r} d W_{r}+\int_{s}^{t} Y_{r}^{q} d r
$$

where we have used $Z_{r}=0$ for $r \in(\tau, t)$, which finishes the proof that $(Y, Z)$ satisfies (3). 


\subsection{Solution of the heat equation with reaction}

This subsection proves the key ingredient of Theorem 1, i.e., the existence of a classical solution $u$ of (10) and the boundary condition (13). Equation (10) is often referred to as a reaction-diffusion equation where $V^{q}$ is the reaction term [7, Example 1, page 535]. The main difficulty with (10) and (13) is the discontinuity and unboundedness of the boundary condition near the corners $(L, T)$ and $(0, T)$ in $\mathbb{R}^{2}$. The next proposition asserts the existence of $u$ and gives its regularity properties (the function $v_{0}$ is defined in (18)). Define

$$
v_{0}(x, t) \doteq \mathbb{E}_{x, t}\left[y_{\tau} \mathbf{1}_{\{\tau<T\}}\right],
$$

where the subscript $(x, t)$ of the expectation operator denotes conditioning on $W_{t}=x$. The function $v_{0}$ will play a key role in our construction of the solution $u$.

Proposition 2. There is a unique function $0 \leq u \leq v_{0}$ which is $C^{\infty}$ in the $x$ variable and continuously differentiable in the $t$ variable over $D$ and is continuous on $\bar{D} \backslash\{(L, T),(0, T)\}$ and which solves (10) and (13).

An intermediate step in the proof of Proposition 2 will be to show that $v_{0}$ of (18) solves the classical heat equation

$$
\partial_{t} V+\frac{1}{2} \partial_{x x} V=0
$$

over $D=(0, L) \times(0, T)$, with the same boundary condition (13) (see subsection 2.2.1 below). In this, the assumption $q>2$ and the following fact will play a key role: $q>2$ implies that $-1<1-p<0$ and thus the solution (2) is integrable:

$$
\int_{0}^{T} y_{s} d s<\infty
$$

Following notation parallel to that of [8] define

$$
B_{t_{0}} \doteq\left\{(x, t), x \in(0, L), t=t_{0}\right\}, \quad B \doteq\{(x, t), x \in(0, L), t=T\}, \quad S \doteq \partial D \backslash\left\{B_{0} \cup \bar{B}\right\} ;
$$

these sets are depicted in Figure 1. The proof of Proposition 2 will proceed as follows:

1. $q>2$ implies that $v_{0}$ solves (in the classical sense) the linear heat equation (19) and the boundary condition (13) (see Lemmas 1 and 2 in subsection 2.2.1 below),

2. Approximate (13) by a sequence of smooth boundary conditions to which standard classical PDE theory applies and yields classical solutions. The solutions of the approximating equations are monotone in the approximation parameter, and their limit is our candidate solution $u$. Itô's formula implies an expectation representation for the approximate solutions. The solution $v_{0}$ of the heat equation in the first step gives us the necessary bound to invoke the dominated convergence theorem to infer that $u$ satisfies the same expected value representation as the prelimit functions (see Lemma 4 and (29)).

3. Establish the regularity properties of $u$ (see Lemma 5); we do this in two different ways. The first approach relies only on probabilistic arguments and is elementary and direct, it uses the following elements: a) explicit formulas for the density of the hitting time $\tau=\inf \left\{t: W_{t} \in\{0, L\}\right\}$ and the density of $W_{t}$ over sample paths restricted to stay in the interval $(0, L)$ upto time $t \mathrm{~b})$ Duhamel's principle and $\mathrm{c}$ ) the expected value representation of $u$. The second approach is based on analytic arguments for parabolic uniformly elliptic PDE. 


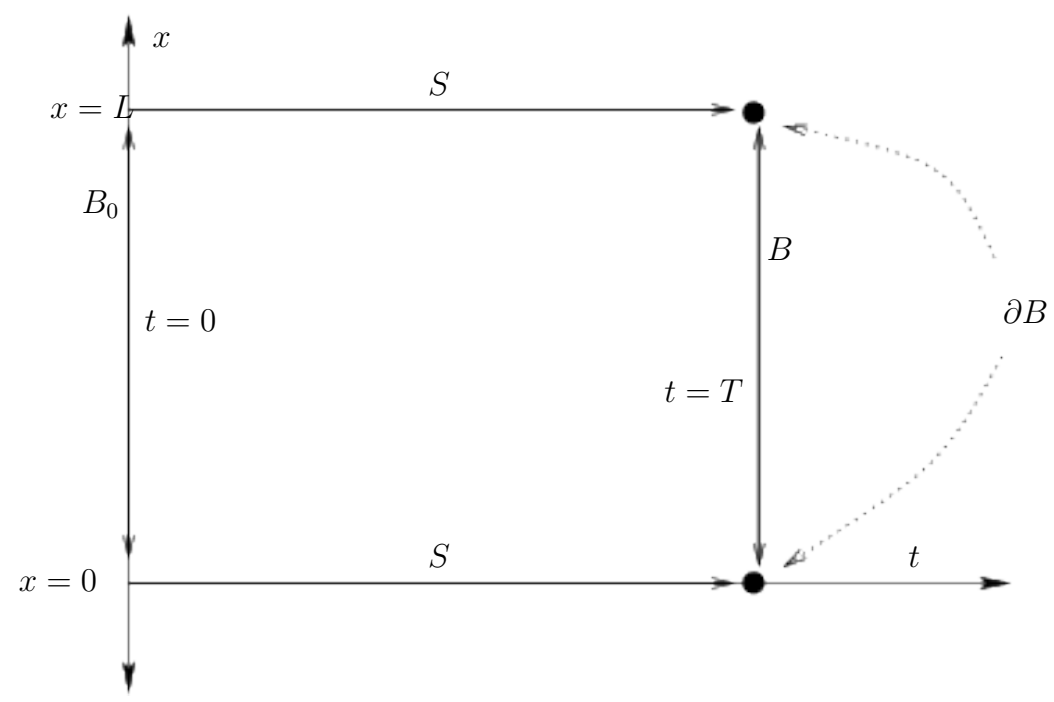

Figure 1: The domain and its boundaries

4. Establish the continuity properties of $u$ (Lemma 6),

5. Once enough regularity is proved, the proof that $u$ actually solves the PDE follows from Itô's formula, the expectation representation of $u$ and the strong Markov property of $W$.

The above elements are put together in the Proof of Proposition 2 given at the end of subsection 2.2.2.

\subsubsection{Solution of the classical heat equation with singularities at the corners}

The classical theory of Brownian motion and of the classical heat equation suggest that $v_{0}$ is the unique solution of (19) and the boundary condition (13). Let us prove that $v_{0}$ is finite and that it indeed solves (19) and (13). Equation [5, (4.1)] (or Itô's formula and direct computation) implies the following formula for the distribution function of $\tau$ conditioned on $W_{t}=x$ :

$$
\mathbb{P}_{x, t}(\tau \leq s)=1+\mathbb{P}_{x, 0}\left(W_{s-t} \in A^{c}\right)-\mathbb{P}_{x, 0}\left(W_{s-t} \in A\right)
$$

where $A \doteq \cup_{n \in \mathbb{Z}}\{2 n L+[0, L]\}, x \in[0, L]$ and $s>t$. Substitute $A$ in (21) and change variables to rewrite $(21)$ as

$$
\mathbb{P}_{x, t}(\tau \leq s)=1+\sum_{n \in \mathbb{Z}} \frac{1}{\sqrt{2 \pi}}\left(\int_{\frac{(2 n+1) L-x}{\sqrt{s-t}}}^{\frac{(2 n+2) L-x}{\sqrt{s-t}}} e^{-y^{2} / 2} d y-\int_{\frac{2 n L-x}{\sqrt{s-t}}}^{\frac{(2 n+1) L-x}{\sqrt{s-t}}} e^{-y^{2} / 2} d y\right) .
$$

For $x \in(0, L)$, the derivative of the last display with respect to $s$ gives the density of $\tau$ :

$$
f_{\tau}(x, t, s) \doteq \frac{(s-t)^{-3 / 2}}{\sqrt{2 \pi}} \sum_{n \in \mathbb{Z}}((2 n+1) L-x) e^{-\frac{((2 n+1) L-x)^{2}}{2(s-t)}}-(2 n L-x) e^{-\frac{(2 n L-x)^{2}}{2(s-t)}}
$$

(for $x \in\{0, L\}, \tau=t$ and $\mathbb{P}_{x, t}(\tau>s)=0$ identically for $s>t$ and indeed the right side of (21) is identically 0 for $x \in\{0, L\})$; Figure 2 shows the graph of $f_{\tau}$ for $t=2, L=4, x=3.5$ 


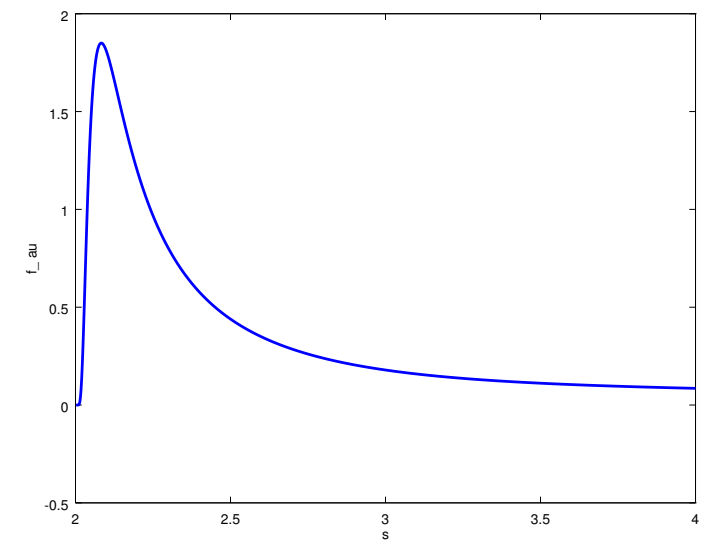

Figure 2: The graph of $f_{\tau}, t=2, L=4, x=3.5$

For $(x, t) \in D$, write the expectation in (18) in terms of the density $f_{\tau}$ :

$$
v_{0}(x, t)=\int_{t}^{T} f_{\tau}(x, t, s) y_{s} d s=\int_{t}^{T} f_{\tau}(x, t, s)((q-1)(T-s))^{1-p} d s .
$$

The formula (22) and the behavior of $x \log (x)$ around 0 imply that $f_{\tau}$ is continuous and smooth with continuous derivatives over the region $\left[\delta_{1}, L-\delta_{2}\right] \times[t, \infty)$ for any $\delta_{i}>0$ with $\delta_{1}<L-\delta_{2}$. Therefore from (20) we deduce that $v_{0}(x, t)<\infty$ for $(x, t) \in D$ and $v_{0}$ has the same regularity as $f_{\tau}$ in compact subsets of $D$. Integrability of $t \mapsto y_{t}(20)$, the boundedness of $f_{\tau}$ in compact subsets of $D,(23)$ and the dominated convergence theorem also imply $v_{0}(x, t) \rightarrow 0$ for $x \in(0, L)$ and $t \rightarrow T$. Furthermore, for any $t<T, f_{\tau}$ is continuous as a function of $(x, s)$ on any compact strip $[0, L] \times[T-\delta, T]$ as long as $t<T-\delta$. This and (20) imply

$$
\left|v_{0}(x, t)-\int_{t}^{T-\delta} f_{\tau}(x, t, s) y_{s} d s\right| \leq \epsilon
$$

for any $\epsilon>0$ when $\delta>0$ is small enough. Note

$$
\int_{t}^{T-\delta} f_{\tau}(x, t, s) y_{s} d s=\mathbb{E}_{x, t}\left[y_{\tau} \mathbf{1}_{\{\tau<T-\delta\}}\right] .
$$

$\mathbb{P}_{x, t}(\tau=T-\delta)=0$, and $s \mapsto y_{s} \mathbf{1}_{\{s<T-\delta\}}$ is a continuous and bounded function for $s \neq T-\delta$. Now choose any sequence $\left(t_{n}, x_{n}\right) \rightarrow(t, x), x \in\{0, L\}$ and $t<T$. The law of the iterated logarithm [15, Theorem 9.23] implies that the hitting time $\tau$ converges to $t$ as $n \rightarrow \infty$. These imply

$$
\lim _{n \rightarrow \infty} \mathbb{E}_{\left(x_{n}, t_{n}\right)}\left[y_{\tau} \mathbf{1}_{\{\tau<T-\delta\}}\right]=y_{t} .
$$

This and (24) imply $v_{0}\left(x_{n}, t_{n}\right) \rightarrow y_{t}$. Let us record what we have proved so far as a lemma:

Lemma 1. The function $v_{0}$ defined in (18) has the integral representation (23), is smooth in $D$ (with continuous derivatives of all orders in compact subsets of $D$ ) and continuous on $\bar{D} \backslash \partial B$ and satisfies the boundary condition (13).

Next we will use Itô's formula and the regularity of $v_{0}$ to show that in fact it is a solution to the heat equation (19). 
Lemma 2. $v_{0}$ solves (19).

Proof. Suppose there is $\left(x_{0}, t_{0}\right) \in D$ such that

$$
\partial_{t} v_{0}\left(x_{0}, t_{0}\right)+\frac{1}{2} \partial_{x x} v_{0}\left(x_{0}, t_{0}\right) \neq 0 .
$$

Let $\delta>0$, be so that $0<x_{0}-\delta<x_{0}+\delta<L$ and $t_{0}+\delta<T$. By the previous proposition $v_{0}$ is smooth on the compact set $N_{x_{0}} \doteq\left[x_{0}-\delta, x_{0}+\delta\right] \times\left[t_{0}, t_{0}+\delta\right]$ with continuous derivatives of all orders. Let $\tau_{\delta}$ be the first time the process $\left(t, W_{t}\right)$ hits $\partial N_{x_{0}}$. By definition $\tau_{\delta}<\tau \wedge T$. Conditioning on $\mathscr{F}_{\tau_{\delta}}$, the strong Markov property of the Brownian motion and the definition of $v_{0}$ imply

$$
v_{0}\left(x_{0}, t_{0}\right)=\mathbb{E}_{x_{0}, t_{0}}\left[v_{0}\left(W_{\tau_{\delta}}, \tau_{\delta}\right)\right] .
$$

Itô's formula applied to $v_{0}$ up to time $\tau_{\delta}$ gives

$$
\begin{aligned}
& \mathbb{E}_{x_{0}, t_{0}}\left[v_{0}\left(W_{\tau_{\delta}}, \tau_{\delta}\right)\right]-v_{0}\left(x_{0}, t_{0}\right) \\
& \quad=\mathbb{E}_{x_{0}, t_{0}}\left[\int_{t}^{\tau_{\delta}}\left(\partial_{t} v_{0}\left(W_{s}, s\right)+\frac{1}{2} \partial_{x x} v_{0}\left(W_{s}, s\right)\right) d s\right] .
\end{aligned}
$$

(26) implies that the left side of the last display equals 0 . But the continuity of $\partial_{t} v_{0}+\frac{1}{2} \partial_{x x} v_{0}$ on $N_{x_{0}}, \tau_{\delta} \neq 0$ and (25) imply that the right side of (27) is nonzero, which is a contradiction. Hence, (25) cannot happen and $v_{0}$ indeed solves (19) in $D$.

Note that $v_{0}$ is the unique function satisfying the properties of Lemma 1 and Lemma 2. This follows from the arguments of the previous proof or from [17, Theorem III.5.1].

\subsubsection{Treating the $V^{q}$ term}

Equipped with the classical solution $v_{0}$ of the heat equation (19) and the boundary condition (13) we will proceed as follows to construct a classical solution to (10) and (13): define a family of boundary conditions $y^{m, n}$ approximating $y$ (decreasing in $m$ and increasing in $n$ ) which are smooth upto $\partial D$ satisfying the existence uniqueness results from the classical theory of parabolic PDE [8]. This gives us a family of functions $u_{m, n}$, solving (10) with boundary values $y^{m, n}$ and which, by Itô's formula, have expected cost representations. This, the dominated convergence theorem and (20) give, upon taking limits of $\left\{u_{m, n}\right\}$, a candidate solution $u$, which also has the same expected cost representation as the prelimit functions $u_{m, n}$. We will then use the expected cost representation of $u$ to improve our knowledge of $u$ 's regularity.

The next lemma is a consequence of the maximum principle ${ }^{2}$ and is well known for BSDE with monotone generator.

\section{Lemma 3.}

1. Suppose $u_{0} \geq 0$ and $u_{1} \geq 0$ are two bounded smooth solutions of (10) such that $\left.u_{0}\right|_{\partial D \backslash B_{0}} \geq\left. u_{1}\right|_{\partial D \backslash B_{0}}$. Then $u_{0} \geq u_{1}$ on $D$.

2. Assume that $u_{0}$ is a continuous solution of (10) with $\left|u_{0}\right| \leq K$ on $\partial D \backslash B_{0}$. Then $\left|u_{0}\right| \leq K$ on $\bar{D}$.

\footnotetext{
${ }^{2}$ The maximum principle also holds under much weaker assumptions (see among others Lemma 2.7 in [19] or Lemma 1.6 in $[20])$.
} 
Proof. $v=\left(u_{0}-u_{1}\right)$ satisfies

$$
\partial_{t} v+\frac{1}{2} \partial_{x x} v-\frac{1}{2} R v=0
$$

where $R=\left(u_{0}^{q}-u_{1}^{q}\right) /\left(u_{0}-u_{1}\right) \mathbf{1}_{u_{0} \neq u_{1}}>0$ and the boundary condition $u_{0}-u_{1} \geq 0$ on $\partial D \backslash B_{0}$. Itô's formula implies

$$
v(x, t)=\mathbb{E}_{x, t}\left[e^{-\int_{t}^{\tau \wedge T} R\left(W_{s}, s\right) d s} v\left(W_{\tau \wedge T}, \tau \wedge T\right)\right] \geq 0 .
$$

For the second claim of the lemma, we use the same estimate with $u_{1}=0$ :

$$
u_{0}(x, t)=\mathbb{E}_{x, t}\left[e^{-\int_{t}^{\tau \wedge T} R\left(W_{s}, s\right) d s} u_{0}\left(W_{\tau \wedge T}, \tau \wedge T\right)\right] .
$$

The next lemma identifies our candidate solution to the PDE (19) and the boundary condition (13).

Lemma 4. There exists a measurable function $0 \leq u \leq v_{0}$ which satisfies

$$
u(x, t)=\mathbb{E}_{x, t}\left[e^{-\int_{t}^{\tau} u^{q-1}\left(W_{s}, s\right) d s} y_{\tau} \mathbf{1}_{\{\tau<T\}}\right]
$$

or equivalently

$$
u(x, t)=\mathbb{E}_{x, t}\left[-\int_{t}^{\tau \wedge T} u^{q}\left(W_{s}, s\right) d s+y_{\tau} \mathbf{1}_{\{\tau<T\}}\right]
$$

for $(x, t) \in D$.

Proof. Define

$$
y_{t}^{(n)} \doteq y_{t-1 / n} .
$$

Hence for any $t \in[0, T],\left|y_{t}^{(n)}\right| \leq\left((q-1)^{1-p}\right) n^{p-1}$. Define $\psi: \partial D \backslash B \rightarrow \mathbb{R}$ and $\psi_{n}: \partial D \backslash B \rightarrow \mathbb{R}$ as follows:

$$
\begin{aligned}
\psi(x, T) & =\psi_{n}(x, T)=0, x \in(0, L), \\
\psi(x, t) & =y_{t},(x, t) \in S, \\
\psi_{n}(x, t) & =y_{t}^{(n)},(x, t) \in S .
\end{aligned}
$$

The function $\psi$ describes exactly the boundary condition (13). Note that $\psi$ and $\psi_{n}$ are discontinuous at the corners $\partial B$ and $\psi_{n} \nearrow \psi$. We will now approximate $\psi_{n}$ by a sequence of smooth $\psi_{m, n}$ so that we can invoke [8, Theorem 9, page 205]. This result requires that $\psi_{m, n} \in \bar{C}^{2+\delta}$ for $\delta \in(\alpha, 1)$, where $\alpha$ is the Hölder constant associated with the boundary $S$, and

$$
\partial_{t} \psi_{m, n}+\frac{1}{2} \partial_{x x} \psi_{m, n}-\psi_{m, n}^{q}=0
$$

on $\partial B$.

To get the desired sequence, begin with two functions (linear in $x$ ):

$$
\begin{aligned}
& \psi_{m, n}^{(0)}(x, t) \doteq y_{t}^{(n)}[1-x m / 2], \\
& \psi_{m, n}^{(L)}(x, t) \doteq y_{t}^{(n)}[1-(L-x) m / 2] .
\end{aligned}
$$


Let $\eta: \mathbb{R} \rightarrow[0,1], \eta \in C^{\infty}$ be as follows: $\eta^{\prime}<0$ on $(0,1), \eta \geq 0, \eta(x)=1$ for $x \leq 0, \eta(x)=0$, for $x>1$; one possible choice is

$$
\eta(x)=\int_{(x \vee 0) \wedge 1}^{1} e^{\frac{-1}{1-(2 y-1)^{2}}} d y / \int_{0}^{1} e^{\frac{-1}{1-(2 y-1)^{2}}} d y .
$$

Now define

$$
\psi_{m, n}(x, t)=\psi_{m, n}^{(0)}(x, t) \eta\left(\frac{m^{2} x-1}{m-1}\right)+\psi_{m, n}^{(L)}(x, t) \eta\left(\frac{m^{2}(L-x)-1}{m-1}\right) ;
$$

for $m>2 / L \vee 1$. The resulting sequence $\psi_{m, n}$ of functions are nonnegative and smooth, decreasing in $m$ with limit $\psi_{n}$ and they all satisfy (31). Figure 3 shows the graph of $\psi_{m, n}$ for $m=5, n=10, L=3$ and $T=1$.

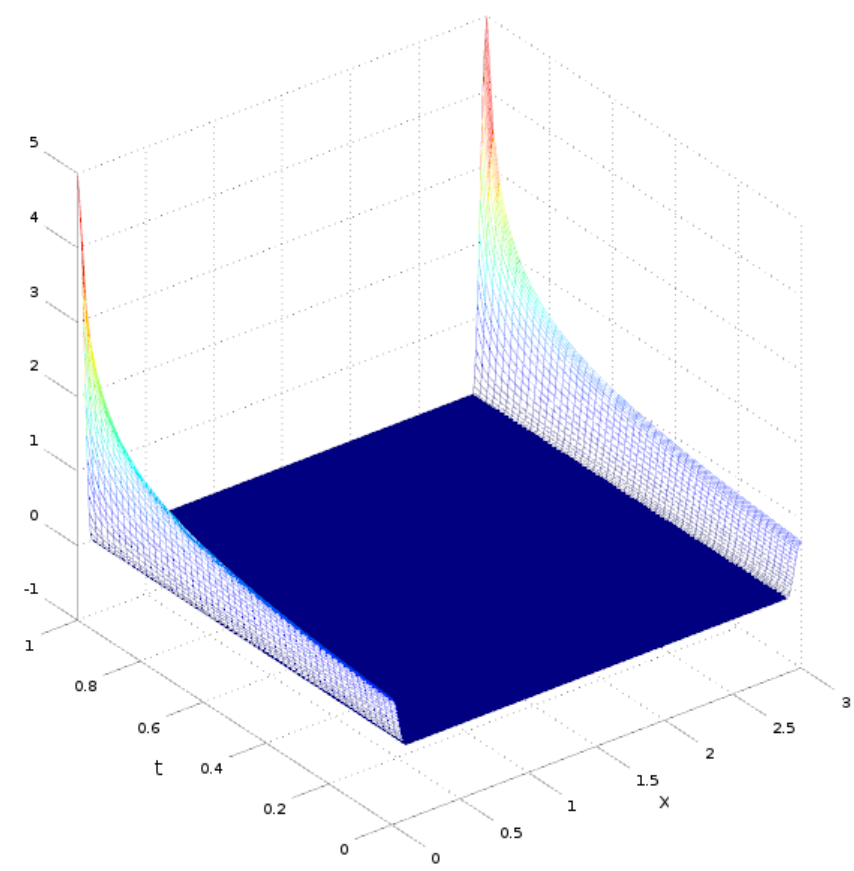

Figure 3: $\quad \psi_{m, n}, m=10, n=50, L=3, T=1$

Now we consider the PDE (10) with the boundary condition

$$
\left.V\right|_{\partial D \backslash B_{0}}=\psi_{m, n} .
$$

$\left|\psi_{m, n}\right| \leq\left((q-1)^{1-p}\right) n^{p-1}=\gamma_{n, q}$ implies that it suffices for our purposes to look for a solution $\left|u_{m, n}\right| \leq \gamma_{n, q}$ (see Lemma 3). The $f(x) \doteq x^{q}$ term in (10) is continuous in the interval $\left[0, \gamma_{n, q}\right]$ and in particular it satisfies [8, equation (4.10), page 203]

$$
x f(x) \leq A_{1} x^{2}+A_{2}, \quad x \in\left[0, \gamma_{n, q}\right],
$$

for constants $A_{1}, A_{2} \geq 0$, and being monotone itself, it trivially satisfies [8, equation (4.17), page 205], which requires $f$ be bounded by a monotone function. These imply that $[8$, 
Theorem 9, page 205] is applicable, and therefore, the PDE (10) and the boundary condition (32) have a classical solution $u_{m, n}$ that is continuous in $\bar{D}$. Itô's formula gives

$$
\begin{aligned}
u_{m, n}(x, t) & =\mathbb{E}_{x, t}\left[e^{-\int_{t}^{\tau \wedge T} u_{m, n}^{q-1}\left(W_{s}, s\right) d s} \psi_{m, n}\left(W_{\tau \wedge T}, \tau \wedge T\right)\right] \\
& =\mathbb{E}_{x, t}\left[-\int_{t}^{\tau \wedge T} u_{m, n}^{q}\left(W_{s}, s\right) d s+\psi_{m, n}\left(W_{\tau \wedge T}, \tau \wedge T\right)\right] .
\end{aligned}
$$

$\psi_{m, n} \geq 0$ and Lemma 3 imply $u_{m, n} \geq 0$. The functions $\psi_{m, n}$ are decreasing in $m$ and increasing in $n$ and they are all bounded; this and Lemma 3 imply that $u_{m, n}$ is decreasing in $m$ and increasing in $n$. Then we can define

$$
0 \leq u_{n} \doteq \lim _{m \rightarrow \infty} u_{m, n}
$$

and

$$
0 \leq u \doteq \lim _{n \rightarrow \infty} u_{n}
$$

The representation (33), $u_{m, n} \geq 0, \psi_{m, n} \leq y_{t}$, and the definition (18) of $v_{0}$ imply $u_{m, n} \leq v_{0}$. Then by the above definitions $u \leq v_{0}$. Now the dominated convergence theorem (where $y_{\tau} \mathbf{1}_{\{\tau<T\}}$ serves as the dominating function), $v_{0}<\infty$, and taking limits in (33) give

$$
\begin{aligned}
u(x, t) & =\mathbb{E}_{x, t}\left[e^{-\int_{t}^{\tau \wedge T} u^{q-1}\left(W_{s}, s\right) d s} y_{\tau} \mathbf{1}_{\{\tau<T\}}\right] \\
& =\mathbb{E}_{x, t}\left[-\int_{t}^{\tau \wedge T} u^{q}\left(W_{s}, s\right) d s+y_{\tau} \mathbf{1}_{\{\tau<T\}}\right] .
\end{aligned}
$$

Our next task is to establish that $u$ is smooth in $D$.

Lemma 5. The function $u$ of (29) is $C^{\infty}$ in $x$ and continuously differentiable in $t$ over $D$.

We will give two different proofs for Lemma 5. The first is based on Duhamel's principle and uses the density of $W_{s}, s>t$ on $[0, L]$ over the set $\left\{0<W_{u}<L, \forall u \in[t, s]\right\}$; this proof is based on fairly elementary calculations and in that sense direct. We will define a number of functions $\left(U_{1}, U_{2}, U_{3}\right.$ and $\left.U_{4}\right)$ in this proof, which will also be used in the proof of the continuity of $u$ on $\bar{D} \backslash \partial B$ in Lemma 6 below. The second proof uses general analytic results on the solution of uniformly elliptic parabolic PDE.

For the first proof we need the density $\mathbb{P}\left(W_{s} \in d x, \tau>s\right)$ whose formula is given as [5, Equation (4.1)]; let us rederive it using our notation. Parallel to (21) one first writes

$$
\begin{aligned}
& \mathbb{P}_{x, t}\left(W_{s} \in(0, a), 0<W_{u}<L, u \in(t, s)\right) \\
& \quad=\mathbb{P}_{x, 0}\left(W_{s-t} \in A_{a}\right)-\mathbb{P}_{x, 0}\left(W_{s-t} \in B_{a}\right),
\end{aligned}
$$

for $a \in(0, L)$ and where $A_{a} \doteq \cup_{n \in \mathbb{Z}}\{2 n L+(0, a)\}$ and $B_{a} \doteq \cup_{n \in \mathbb{Z}}\{2 n L+(2 L-a, 2 L)\}$; the identities $A_{L}=A$ and $B_{L}=A_{L}^{c}=A^{c}$ imply that (21) is a special case of (34). Substituting $A_{a}$ and $B_{a}$ in (34) and the normal distribution of $W_{s-t}$ give

$$
\begin{aligned}
& \mathbb{P}_{x, t}\left(W_{s} \in(0, a), 0<W_{u}<L, u \in(t, s)\right) \\
& \quad=\sum_{n \in \mathbb{Z}} \frac{1}{\sqrt{2 \pi}}\left(\int_{\frac{2 n L-x}{\sqrt{s-t}}}^{\frac{a+2 n L-x}{\sqrt{s-t}}} e^{-y^{2} / 2} d y-\int_{\frac{2 n L-a-x}{\sqrt{s-t}}}^{\frac{2 n L-x}{\sqrt{s-t}}} e^{-y^{2} / 2} d y\right) .
\end{aligned}
$$


Differentiate the last display to get the density of $W_{s}$ on $(0, a)$ when the sample path of $W$ is constrained to stay in $[0, L]$ over the time interval $[t, s]$ :

$$
f_{W}(x, t, s) \doteq \frac{1}{\sqrt{2 \pi(s-t)}} \sum_{n \in \mathbb{Z}}\left(e^{\frac{-(a+2 n L-x)^{2}}{2(s-t)}}-e^{\frac{-(2 n L-a-x)^{2}}{2(s-t)}}\right) .
$$

The above display implies that $f_{W}$ is smooth for $s>t$ and $x \in(0, L)$ in all variables with continuous derivatives of all orders. Now we proceed with the first proof of Lemma 5.

Proof. Write $u$ as the sum

$$
u(x, t)=-U_{1}(x, t)+v_{0}(x, t)
$$

where

$$
U_{1}(x, t) \doteq \mathbb{E}_{x, t}\left[\int_{t}^{\tau \wedge T} u^{q}\left(W_{s}, s\right) d s\right], \quad v_{0}(x, t)=\mathbb{E}_{x, t}\left[y_{\tau} \mathbf{1}_{\{\tau<T\}}\right]
$$

We already know that $v_{0}$ satisfies the conditions listed in the proposition. It rests to show the same for $U_{1}$. First, $0 \leq u \leq v_{0}$ implies

$$
0 \leq U_{1} \leq v_{0}
$$

Fix an arbitrary $T>\delta>0$. We will now show that $U_{1}$ is smooth in $(0, L) \times(0, T-\delta), \delta$ being arbitrary, this will show $U_{1}$ is smooth on $(0, L) \times(0, T)$. For $t<T-\delta$, the strong Markov property of $W$ and conditioning on $\mathscr{F}_{\tau \wedge(T-\delta)}$ imply that we can write $U_{1}$ in two pieces as follows:

$$
\begin{aligned}
U_{1}(x, t) & =\mathbb{E}_{x, t}\left[\int_{t}^{\tau \wedge(T-\delta)} u^{q}\left(W_{s}, s\right) d s+U_{1}\left(W_{T-\delta}, T-\delta\right) \mathbf{1}_{\{\tau>T-\delta\}}\right] \\
& =U_{2}(x, t)+U_{4}(x, t),
\end{aligned}
$$

where

$$
\begin{aligned}
U_{2}(x, t) & \doteq \mathbb{E}_{x, t}\left[\int_{t}^{\tau \wedge(T-\delta)} u^{q}\left(W_{s}, s\right) d s\right], \\
U_{4}(x, t) & \doteq \mathbb{E}_{x, t}\left[U_{1}\left(W_{T-\delta}, T-\delta\right) \mathbf{1}_{\{\tau>T-\delta\}}\right]
\end{aligned}
$$

Let us write $U_{4}$ using the density $f_{W}$ given in (35):

$$
U_{4}(x, t)=\int_{0}^{L} f_{W}(x, t, T-\delta, y) U_{1}(y, T-\delta) d y .
$$

That $0 \leq v_{0}$ is continuous on $\bar{D} \backslash \partial B$ implies that it is in particular bounded on $[0, L] \times$ $[0, T-\delta]$. This and (37) imply that $U_{1}(\cdot, T-\delta)$ is bounded by the same bound. This, the existence and the continuity of the derivatives of $f_{W}$ in $x$ and $t$ imply that $U_{4}$ is smooth in $(0, L) \times[0, T-\delta]$ and is continuous on $[0, L] \times[0, T-\delta)$. To study $U_{2}$ we will use Duhamel's principle:

$$
U_{2}(x, t)=\mathbb{E}_{x, t}\left[\int_{t}^{\tau \wedge(T-\delta)} u^{q}\left(W_{s}, s\right) d s\right]=\mathbb{E}_{x, t}\left[\int_{t}^{T-\delta} \mathbf{1}_{\{s<\tau\}} u^{q}\left(W_{s}, s\right) d s\right] .
$$


$v_{0} \geq u \geq 0$ and Fubini's theorem imply

$$
U_{2}(x, t)=\int_{t}^{T-\delta} \mathbb{E}_{x, t}\left[\mathbf{1}_{\{s<\tau\}} u^{q}\left(W_{s}, s\right)\right] d s .
$$

Define

$$
U_{3}(x, t, s) \doteq \mathbb{E}_{x, t}\left[\mathbf{1}_{\{s<\tau\}} u^{q}\left(W_{s}, s\right)\right]
$$

and write (39) in terms of $U_{3}$ (this is Duhamel's principle):

$$
U_{2}(x, t)=\int_{t}^{T-\delta} U_{3}(x, t, s) d s .
$$

The function $U_{3}$ can be written in terms of the density $f_{W}$ as

$$
U_{3}(x, t, s)=\int_{0}^{L} f_{W}(x, t, s, y) u^{q}(y, s) d y .
$$

Once again, for $s<T-\delta, 0 \leq u^{q}$ is uniformly bounded above by a constant. This, the smoothness of $f_{W}$ in $x$ imply that $U_{3}$ is smooth in $x$ and $t$ on $(0, L) \times(0, T-\delta)$ for $t<s$. $U_{3}$ is smooth in its $x$ variable, therefore $U_{2}$ is also smooth in $x$ over the region $(0, L) \times(0, T-\delta)$. This, the smoothness of $U_{4}$ and (38) imply the same for $U_{1}$; the smoothness of $U_{1}$ in $x$ and (36) imply the smoothness of $u$ in $x$.

Now we will derive the regularity of $u$ in the $t$ variable. Let us begin with continuity of $U_{2}$ in $t$ : take any sequence $t_{n} \rightarrow t$, with $0<t_{n}, t<T-\delta$ and $x \in(0, L)$. The continuity of $U_{3}$ in the $t$ variable implies that the sequence of functions

$$
s \mapsto \mathbf{1}_{\left\{s>t_{n}\right\}} U_{3}\left(x, t_{n}, s\right)
$$

converge almost surely to

$$
s \mapsto \mathbf{1}_{\{s>t\}} U_{3}(x, t, s)
$$

on the set $(0, T-\delta)$. This and the bounded convergence theorem imply

$$
U_{2}\left(x, t_{n}\right) \rightarrow U_{2}(x, t),
$$

i.e., $U_{2}$ is also continuous in the $t$ variable on the set $(0, L) \times(0, T-\delta)$. Thus we have: $U_{2}$, $U_{4}$ are both continuous on $(0, L) \times(0, T-\delta)$. This and (38) imply that $U_{1}$ is continuous over the same domain, this and (36) imply the same for $u$. Now going back to (41) we see that this implies that $U_{3}$ is also continuous in the $s$ variable. The continuity of $U_{3}$ in all of its variables, (40) and the fundamental theorem of calculus tell us that $U_{2}$ is differentiable in $t$ and

$$
\begin{aligned}
\frac{\partial U_{2}}{\partial t} & =-U_{3}(x, t, t)+\int_{t}^{T-\delta} \frac{\partial U_{3}}{\partial t}(x, t, s) d s \\
& =-u^{q}(x, t)+\int_{t}^{T-\delta} \frac{\partial U_{3}}{\partial t}(x, t, s) d s,
\end{aligned}
$$

which, in particular, is a continuous function on $(0, L) \times(0, T-\delta)$. Finally, this, the regularity of $U_{4}$ and (38) imply that $U_{1}$ is differentiable in $t$ with continuous derivative over the domain $(0, L) \times(0, T-\delta)$, which in its turn, along with (36) imply the same for $u$. This finishes the smoothness claims of the lemma on $u$. 
We now give an alternative proof of the same lemma using classical but deep results on parabolic PDE with a regularization bootstrap argument. We know from Lemma 4 that $u$, by construction, is the limit of a sequence $u_{m, n}$ of classical solutions of the PDE (10) with the boundary condition (32). The comparison principle (Lemma 3) implies that for any $m, n$ we have: $0 \leq u_{m, n}(x, t) \leq y_{t}$ on $[0, L] \times[0, T]$. Thus the solutions are bounded from above by a function independent of $n$ and $m$. This will be useful in the proof below.

Second proof of Lemma 5. Now fix $\epsilon>0$. On $[0, L] \times[0, T-\epsilon], u_{m, n}$ is bounded (uniformly in $n$ and $m)$ by $y_{T-\epsilon}$. Moreover the smooth function $u_{m, n}$ satisfies on $(0, L) \times(0, T-\epsilon)$

$$
\partial_{t} u_{m, n}+\frac{1}{2} \partial_{x x} u_{m, n}=\left(u_{m, n}\right)^{q}=f_{m, n}
$$

with the Hölder continuous lateral boundary condition $y_{t}^{(n)}$ (and a bounded terminal condition $\left.u_{m, n}(x, T-\epsilon)\right)$. Here $f_{m, n}$ is a bounded function. We can apply [17, Theorem III.10.1] (Conditions (1.2) and (7.1) of [17] are trivially satisfied in our setting). Therefore for any $\eta>0, u_{m, n}$ is in $H^{\alpha, \alpha / 2}([\eta, T-\eta] \times[0, T-\epsilon])$ (space of functions which $\alpha$-Hölder continuous in the space variable $x$ and $\alpha / 2$-Hölder continuous in the times variable $t$ ). The value of $\alpha>0$ and the Hölder norm of $u_{m, n}$ does not depend on $m$ and on $n$. In other words the Hölder norm of $u_{m, n}$ is bounded by some constant $C_{\alpha}$ depending only on $\eta$ and $\epsilon$. Moreover we already know that $u_{m, n}$ converges pointwise to $u_{n}$ (as $m$ goes to $+\infty$ ) and $u_{n}$ converges to $u$ (when $n$ tends to $+\infty)^{3}$. Therefore $u_{n}$ and $u$ are in $H^{\alpha, \alpha / 2}([\eta, L-\eta] \times[0, T-\epsilon])$ and their Hölder norms are bounded by the same constant $C_{\alpha}$.

Then $u_{m, n}$ is the solution of the same problem but now with more regular functions $f_{m, n}$ and $u_{m, n}(\cdot, T-\epsilon)$. Thus from [17, Theorem IV.10.1], we know that $u_{m, n}$ is in $H^{2+\alpha, 1+\alpha / 2}([\eta, L-$ $\eta] \times[0, T-\epsilon-\eta])$ for any $\epsilon>0$ and $\eta>0$ and the norm estimates don't depend on $n$ and on $m$, but only on the Hölder norm of $f_{n, m}$ on $[\eta, L-\eta] \times[0, T-\epsilon-\eta]$ and the upper bound on $u_{m, n}$. Thus the same property holds for $u$. In other words $u$ is a classical solution on $(0, L) \times[0, T)$. This regularization argument can be iterated in order to obtain that $u$ is $C^{\infty}$.

Lemma 6. $u$ of (29) is continuous on $\bar{D} \backslash \partial B$.

Proof. Remember that $v_{0}$ is continuous on $B$ and takes the value 0 there. This and $0 \leq u \leq v_{0}$ imply the continuity of $u$ on $B$. By definition $u(x, t)=y_{t}=v_{0}(x, t)$ for $(x, t) \in S$. We already know that $v_{0}$ is continuous on $S$. Furthermore, by definition, $U_{1}=0$ on $S$; these and (36) imply that it suffices to show

$$
U_{1}\left(x_{n}, t_{n}\right) \rightarrow 0
$$

for $\left\{\left(x_{n}, t_{n}\right) \in D\right.$, with $\left(x_{n}, t_{n}\right) \rightarrow(x, t) \in S$. For this, we will use (38) with $\delta>0$ satisfying $t<T-\delta$ and the definitions of $U_{2}$ and $U_{4}$. As $\left(x_{n}, t_{n}\right) \rightarrow(x, t) \in S, \tau \rightarrow t$. This and the boundedness of $u^{q}$ on $[0, L] \times[0, T-\delta]$ implies $U_{2}\left(x_{n}, t_{n}\right) \rightarrow 0$. Lastly, $\tau \rightarrow t$ implies that $\mathbf{1}_{\{\tau>T-\delta\}}$ converges to 0 almost surely. This and the boundedness of $U_{1}$ on over $[0, L] \times$ $[0, T-\delta]$ imply $U_{4}\left(x_{n}, t_{n}\right) \rightarrow 0$. These and (38) establish (42).

We can now complete the proof of Proposition 2:

\footnotetext{
${ }^{3}$ The Arzela-Ascoli theorem implies that $u_{m, n}$ (up to a subsequence) converges to some function $\tilde{u} \in$ $H^{\alpha, \alpha / 2}([\eta, L-\eta] \times[0, T-\epsilon])$. Here $\widetilde{u}=u$ since pointwise convergence has been proved before.
} 
Proof of Proposition 2. By construction $u$ satisfies (13). Lemma 5 says that the function $u$ is smooth on $D$. Thus Itô's formula and the representation formula in Lemma 4 imply that $u$ satisfies (10); the details of a parallel argument have already been given in Lemma 2 and are omitted. Lemma 6 says that $u$ is continuous on $\bar{D} \backslash \partial B ;(42), v_{0}(x, t)=y_{t}$ on $S$ and (36) imply that $u(x, t)=y_{t}$ on $S ; u \leq v_{0}$ and $v_{0}=0$ on $B$ imply $u=0$ on $B$. These imply that $u$ satisfies the boundary condition (13).

Next we prove the uniqueness claim, i.e., if $0 \leq u_{1} \leq v_{0}$ is any other solution of the PDE (19) and the boundary condition (13), continuous on $\bar{D} \backslash \partial B$ then $u_{1}=u$ must hold. Proceeding as in the proof of Lemma 3 define $v=u_{1}-u$ and $R=\left(u_{1}^{q}-u_{0}^{q}\right) /\left(u_{1}-u\right) \mathbf{1}_{u_{1} \neq u}>0$. Now $v$ satisfies the PDE (28), $\left.v\right|_{\partial D}=0$, and is continuous on $\bar{D} \backslash \partial B$. These and Itô's formula imply

$$
v(x, t)=\mathbb{E}_{x, t}\left[e^{-\int_{t}^{T-1 / n} R\left(W_{s}, s\right) d s} v\left(W_{T-\frac{1}{n}}, T-\frac{1}{n}\right) \mathbf{1}_{\{\tau>T-1 / n\}}\right] .
$$

The above display, $R \geq 0,|v| \leq\left|u-u_{1}\right| \leq 2 v_{0}$ and Jensen's inequality imply

$$
|v(x, t)| \leq \mathbb{E}_{x, t}\left[2 v_{0}\left(W_{T-\frac{1}{n}}, T-\frac{1}{n}\right) \mathbf{1}_{\{\tau>T-1 / n\}}\right] .
$$

The expectation representation (18) of $v_{0}$ implies that the second expectation above converges to 0 with $n$. This proves $v=0$.

\subsection{Connection to the minimal super-solution}

It remains to establish the connection between the solution of the BSDE constructed above and the minimal supersolution $\left(Y^{\mathrm{min}}, Z^{\mathrm{min}}\right)$ of the $\operatorname{BSDE}(3,4)$.

Proposition 3. Let $Y$ and $Z$ be the solution of the BSDE (3,4) defined in (15) where for $u$ we take the solution of the PDE (10) and the boundary condition (13) constructed in Proposition 2. Then $(Y, Z)=\left(Y^{\mathrm{min}}, Z^{\mathrm{min}}\right)$.

Proof. It follows from (3) and the definition of the Itô integral that it suffices to prove $Y=Y^{\mathrm{min}}$. The inequality $Y \geq Y^{\mathrm{min}}$ follows from the minimality property (7) of $Y^{\mathrm{min}}$.

To finish the proof we simply need to prove the converse inequality. Recall that $u$ is defined as the limit of a sequence of functions $u_{n}$ (see the proof of Lemma 4). Using the same ideas as used in the proofs of Lemma 5 one can prove that for any $n$, the function $u_{n}$ is smooth and is a classical solution of the PDE (10) in $D$. Moreover, as in the case of $u, u_{n}$ is continuous on $\bar{D} \backslash \partial B$ and satisfies the boundary condition:

$$
u_{n}(0, t)=u_{n}(L, t)=y_{t}^{(n)}=y_{t-1 / n}, t \in[0, T], \quad u_{n}(x, T)=0,0<x<L .
$$

Define

$$
Y_{t}^{n} \doteq\left\{\begin{array} { l l } 
{ u _ { n } ( W _ { t } , t ) } & { , t < \tau \wedge T , } \\
{ y _ { t } ^ { ( n ) } } & { , \tau \leq t \leq T , }
\end{array} \quad Z _ { t } ^ { n } \doteq \left\{\begin{array}{ll}
\partial_{x} u_{n}\left(W_{t}, t\right) & , t<\tau \wedge T \\
0 & , \tau \leq t \leq T .
\end{array}\right.\right.
$$

Straightforward modifications of previous arguments show that $\left(Y^{n}, Z^{n}\right)$ solves the BSDE (3) with terminal condition

$$
\xi^{n}=y_{T}^{(n)} \mathbf{1}_{B(L / 2, L / 2)^{c}}=\left(\frac{n}{q-1}\right)^{\frac{1}{q-1}} \mathbf{1}_{B(L / 2, L / 2)^{c}} .
$$


Since the minimal solution $\left(Y^{\mathrm{min}}, Z^{\mathrm{min}}\right)$ constructed in [23] is the increasing limit of solutions of the same BSDE but with terminal condition $\xi \wedge m$ (as $m$ goes to $+\infty$ ), the comparison principle implies

$$
Y_{t}^{n} \leq Y_{t}^{\min }
$$

a.s. for any $t \in[0, T]$. But $u_{n}$ converges to $u$ and from (15) we obtain the desired inequality.

We finally remark that the solution $(Y, Z)$ of the $\operatorname{BSDE}(3,4)$ (with $\xi=\xi_{1}=\infty$. $\mathbf{1}_{\left.B(L / 2, L / 2)^{c}\right)}$ constructed above is in fact unique. This can be shown using uniqueness results for BSDEs with integrable data provided in [4]. Indeed, for any non-negative solution $(\bar{Y}, \bar{Z})$ of the BSDE with terminal condition $(3,4)$ it holds a.s. that

$$
0 \leq Y_{t}^{\min } \leq \bar{Y}_{t} \leq y_{t}
$$

Hence $\bar{Y}_{t}=y_{t}$ for all $t \in[\tau \wedge T, T]$. In particular, $(\bar{Y}, \bar{Z})$ solves the BSDE on the random time interval $[0, \tau \wedge T]$ and with terminal condition $y_{\tau} 1_{\tau<T}$. Since this terminal condition is in $L^{r}$ for some $r \in(1,1 /(p-1)$ ) (recall that we assume $q>2$ ), it follows from [4, Theorem 5.2 ] that there exists at most one solution $(\bar{Y}, \bar{Z})$ of this BSDE.

\subsection{Numerical examples}

Let us give several numerical examples for the PDE solutions constructed above and the resulting solution $Y$ of the BSDE. The left side of Figure 4 shows the graph of $u_{m, n}$ with $L=3$ and $T=1, m=100$ and $n=50$ computed using a finite difference approximation of the PDE with $\Delta x=0.1$ and $\Delta t=0.01$. The right side of the same figure shows the graph of $u_{m, n}$ over the line $x=L / 2=1.5$ for $m=100$ and $n=10$ and $n=150$ as well as the graph of $y_{t}$; note $u_{100,10}(1.5, t)<u_{100,1000}(1.5, t)<y_{t}$ in the figure, as expected. Figure 5 shows two randomly sampled sample paths of the Brownian motion $W$ with $W_{0}=L / 2=3 / 2$ and the corresponding path for $Y$, computed using (15) where we use a numerical approximation of $u_{m, n}$ with $m=100$ and $n=1000$ to approximate $u$.

\section{The case $\xi=\infty \cdot \mathbf{1}_{B(m, r)}$}

Our results for the terminal condition $\xi=\infty \cdot \mathbf{1}_{B(L / 2, L / 2)}$ parallel those for $\xi=\infty \cdot \mathbf{1}_{B(L / 2, L / 2)^{c}}$, with two differences: 1 ) we need to replace the upperbound $v_{0}$ of subsection 2.2.1 with a new upperbound $\bar{u}$ and 2) $q>1$ is enough for the existence of solutions; see the discussion below for more on these changes.

Theorem 2. Suppose $q>1$. There exists a function $\bar{v}$ which is $C^{\infty}$ in the $x$ variable and $C^{1}$ in the $t$ variable and continuous on $\bar{D} \backslash\{(L, T),(0, T)\}$ and which solves the PDE (10) with the boundary condition (14) (i.e., (44) below)) such that

1. The processes

$$
Y_{t}=\left\{\begin{array}{ll}
\bar{v}\left(W_{t}, t\right) & , t<\tau \wedge T, \\
0 & , \tau \leq t \leq T,
\end{array} \quad Z_{t}= \begin{cases}\bar{v}_{x}\left(W_{t}, t\right) & , t<\tau \wedge T \\
0 & , \tau \leq t \leq T\end{cases}\right.
$$

solve the $B S D E(3,4)$ with $\xi=\infty \cdot \mathbf{1}_{B(L / 2, L / 2)}$, and in particular, $Y$ is continuous on $[0, T]$, 

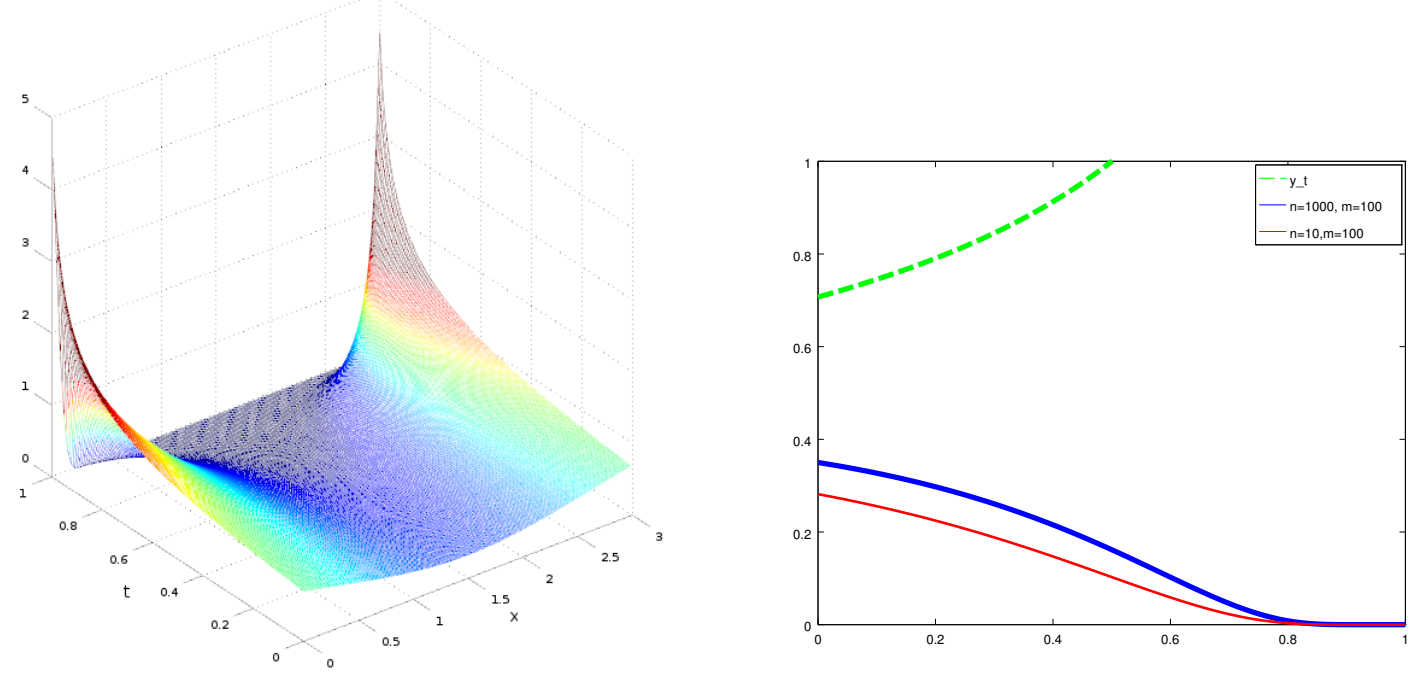

Figure 4: On the left, the graph of $u_{m, n}$ with $m=100$ and $n=50$; on the right, the graph of $u_{m, n}$ over $x=1.5$ for $m=100, n=10$ (thin) and $n=1000$ (thick), and $y_{t}$ (dashed line). In all computations $L=3$ and $T=1$
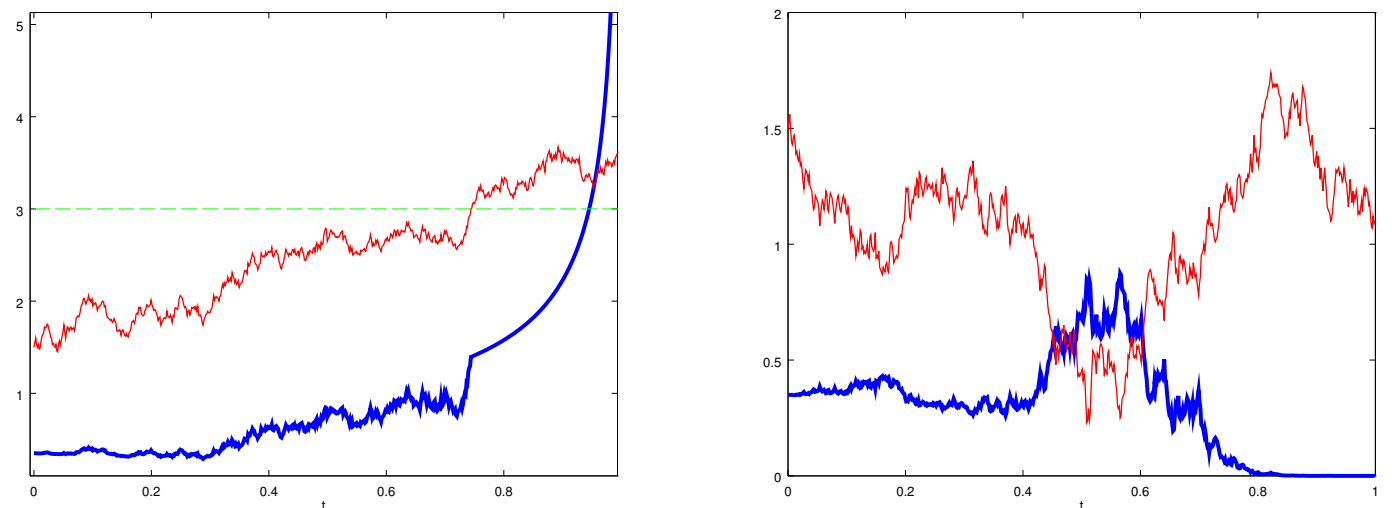

Figure 5: Numerically computed trajectories of $W$ (thin light path) and $Y$ (thick dark) (left with explosion, right without); $Y$ is computed using (15) with $u_{m, n}$ approximating $u$ with $m=100$ and $n=1000 ; L=3$ and $T=1$ 
2. $\left(Y^{\mathrm{min}}, Z^{\mathrm{min}}\right)=(Y, Z)$ and $(8)$ hold.

The steps for the proof of Theorem 2 apply verbatim to the current case except for the construction of the solution of the PDE; for this reason we only give an outline and point out the necessary changes. Breaking as we do in Section 2.1 the BSDE into the intervals $[0, \tau \wedge T]$ and $[\tau \wedge T, T]$, this case can be reduced to the solution of the PDE (10) now with the boundary condition

$$
V(0, t)=V(L, t)=0, t \in[0, T], \quad V(x, T)=\infty, 0<x<L .
$$

The construction given in Section 2.2 for the PDE (10) and the boundary condition (13) allow one to solve the same PDE now with the boundary condition (44) except for the differences pointed out above: in the present case we no longer have the upperbound $v_{0}$ to serve as an upperbound in convergence and continuity arguments. The role of $v_{0}$ will now be played by the limit $\bar{u}$ of a decreasing sequence of solutions of (10). And because we no longer need $v_{0}$ we no longer need the assumption $q>2$ and can work with $q>1$. The details are given in the outline below:

1. First proceed as in Section 2.2, Lemma 4, to construct a classical solution $\bar{u}_{n}$ to (10) on $[0, L] \times[0, T-1 / n]$ with the boundary condition

$$
V(0, t)=V(L, t)=0, t \in[0, T-1 / n], \quad V(x, T-1 / n)=y_{T-2 / n},
$$

$0<x<L$, continuous on $[0, L] \times[0, T-1 / n]-\{(0, T-1 / n),(L, T-1 / n)\}$ satisfying the expectation representations (of type (29) and (30)):

$$
\bar{u}_{n}(x, t)=\mathbb{E}_{x, t}\left[e^{-\int_{t}^{T-1 / n} \bar{u}_{n}^{q-1}\left(W_{s}, s\right) d s} y_{T-2 / n} \mathbf{1}_{\{\tau \geq T-1 / n\}}\right]
$$

or equivalently

$$
\bar{u}_{n}(x, t)=\mathbb{E}_{x, t}\left[-\int_{t}^{\tau \wedge(T-1 / n)} \bar{u}_{n}^{q}\left(W_{s}, s\right) d s+y_{T-2 / n} \mathbf{1}_{\{\tau \geq T-1 / n\}}\right],
$$

for $(x, t) \in[0, L] \times[0, T-1 / n]$.

2. By Lemma 3 for any $(x, t) \in[0, L] \times[0, T-1 / n], \bar{u}_{n}(x, t) \leq y_{t}$. Hence for $n_{1}<n_{2}$ and for any $x \in[0, L], \bar{u}_{n_{2}}\left(x, T-1 / n_{1}\right) \leq y_{T-2 / n_{1}}$. Again by comparison principle (Lemma 3), for any $(x, t) \in[0, L] \times\left[0, T-1 / n_{1}\right], \bar{u}_{n_{2}}(x, t) \leq \bar{u}_{n_{1}}(x, t)$. Then, for fixed $(x, t) \in[0, L] \times[0, T),(x, t)$ will be in the domain of $\bar{u}_{n}(x, t)$ for $n \geq n_{0}$ for some $n_{0}$ and the sequence $\left\{u_{n}(x, t), n \geq n_{0}\right\}$ will be decreasing in $n$; call its limit $\bar{u}(x, t)$, i.e.,

$$
\bar{u}_{n}(x, t) \searrow \bar{u}(x, t), \quad n \rightarrow+\infty .
$$

The representations (45) and (46) and the dominated convergence theorem imply

$$
\begin{aligned}
& \bar{u}(x, t)=\mathbb{E}_{x, t}\left[e^{-\int_{t}^{\tau \wedge r} \bar{u}^{q-1}\left(W_{s}, s\right) d s} \bar{u}\left(W_{r}, r\right)\right], \\
& \bar{u}(x, t)=\mathbb{E}_{x, t}\left[-\int_{t}^{\tau \wedge r} \bar{u}^{q}\left(W_{s}, s\right) d s+\bar{u}\left(W_{r}, r\right)\right],
\end{aligned}
$$

for any $t<r<T$ and any $x \in[0, L]$. Moreover we have

$$
\forall(x, t) \in[0, L] \times[0, T), \quad 0 \leq \bar{u}(x, t) \leq y_{t} .
$$


3. The sufficient differentiability of $\bar{u}$ is proved exactly as in the proofs of Lemma 5 . This implies (via Itô's formula) that $\bar{u}$ solves (10).

4. Next we construct an increasing approximating sequence $\bar{v}_{n}$ by solving the PDE (10) on $[0, L] \times[0, T]$ with the boundary condition

$$
V(0, t)=V(L, t)=0, t \in[0, T], \quad V(x, T)=y_{T-1 / n}, 0<x<L,
$$

and continuous on $\bar{D} \backslash \partial B$.

5. Lemma 3 implies that the sequence $\bar{v}_{n}$ is increasing. Define $\bar{v} \doteq \lim _{n \rightarrow \infty} \bar{v}_{n}$.

6. Lemma 3 and the fact that $(x, t) \mapsto y_{t-1 / n}$ solves (10) imply that $\bar{v}_{n}(\cdot, T-1 / n) \leq$ $y_{T-2 / n}$, which, along with Lemma 3 and the definition of $\bar{u}_{n}$ imply

$$
\bar{v}_{n} \leq \bar{u}_{n}
$$

from which

$$
\bar{v} \leq \bar{u}
$$

follows. Arguments in subsection 2.2 now applied to $\bar{v}$ (with $\bar{u}$ providing the dominating function) imply that $\bar{v}$ has representations of the form (47) and (48), is infinitely differentiable in the $x$ variable and continuously differentiable in the $t$ variable on $(0, L) \times(0, T)$ and that it solves $(10)$.

7. The functions $\bar{v}$ and $\bar{u}$ both satisfy the boundary condition (44) by definition. It remains to show that they are continuous on $\partial D \backslash \partial B$. The continuity of $\bar{u}$ on the lateral boundary $\{(0, t),(L, t), t<T\}$ follows from $0 \leq \bar{u} \leq \bar{u}_{n}$ and the continuity of $\bar{u}_{n}$ on the same boundary. $0 \leq \bar{v} \leq \bar{u}$ implies then the continuity of $\bar{v}$ on the lateral boundary. The continuity of $\bar{v}$ along $B$ follows from the continuity of $\bar{v}_{n}$ along the same boundary and $\bar{v} \geq \bar{v}_{n}$. This and $\bar{u} \geq \bar{v}$ finally imply the continuity of $\bar{u}$ along $B$.

The above algorithm gives us two classical solutions $\bar{u}, \bar{v}$ of the PDE (10) and the boundary condition (44) satisfying $\bar{u} \geq \bar{v}$. In (43) we use the smaller of these solutions to define our solution of the BSDE $(3,4)$ with $\xi=\infty \cdot \mathbf{1}_{B(m, r)}$. That $(Y, Z)$ thus defined satisfies $(3,4)$ as well as the proof of continuity of $Y$ on $[0, T]$ proceed exactly as in the proof of Proposition 1. The proof that $(Y, Z)=\left(Y^{\mathrm{min}}, Z^{\mathrm{min}}\right)$ proceeds as in the proof of Proposition 3 given in subsection 2.3 and follows from $\bar{v}_{n} \nearrow \bar{v}$. In contrast to the setting of Section 2 , the question of uniqueness of $(Y, Z)$ remains open in this case (cf. the remark at the end of Subsection $2.3)$.

We illustrate the computations above with several numerical examples in Figures 6 and 7. The left side of Figure 6 shows the graph of $\bar{u}_{50}$, computed numerically using finite differences; the right side of the same figure shows the graphs of $\bar{u}_{5}(1, t)$ and $\bar{u}_{50}(1, t)$ and $y_{t}$. Figure 7 shows two sets of sample paths of $W$ and $Y$ with $W_{0}=L / 2=1$ and where $Y$ is approximated by $\bar{u}_{50}\left(W_{t}, t\right)$ for $t<\tau$; in all computations $L=2$ and $T=1$.

\section{The Control Interpretation}

We next point out a control interpretation of the BSDE $(3,4)$ or more precisely of the BSDEs $(3,8)$ and $(3,5)$. We consider the case of a general $\mathscr{F}_{T}$-measurable terminal condition $\xi$ possibly taking the value $+\infty$ with positive probability. We use this connection to a stochastic 

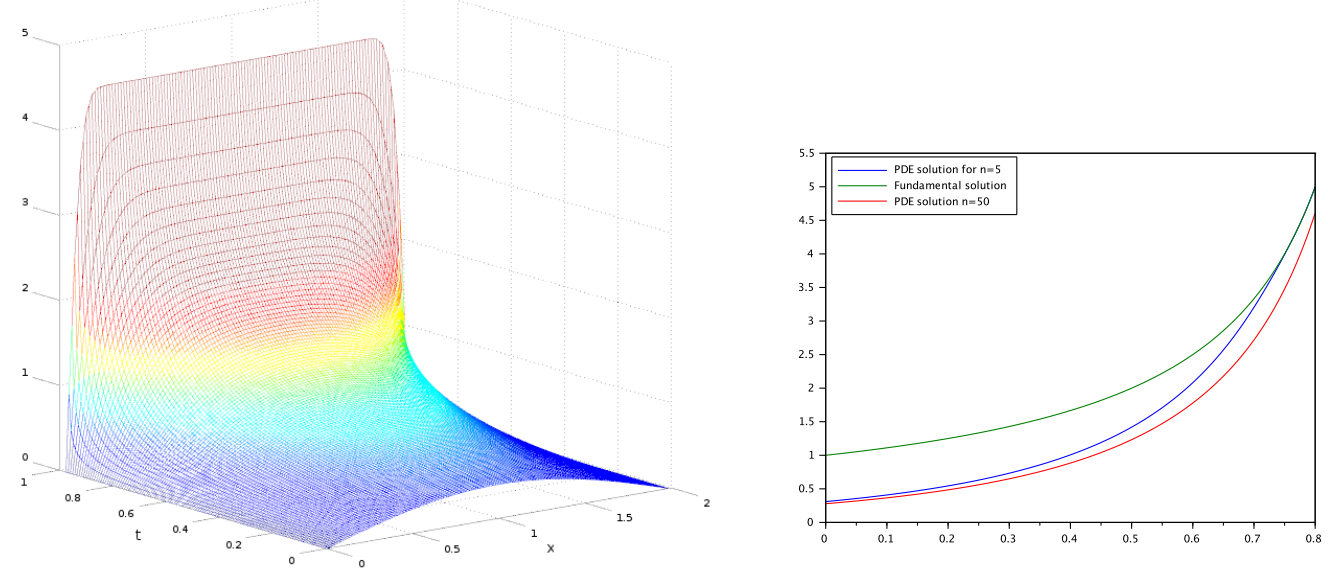

Figure 6: On the left, graph of $\bar{u}_{50}$; on the rights graphs of $\bar{u}_{5}(1, t), \bar{u}_{50}(1, t)$ and $y_{t}$, $t \in[0,1] ; T=1$ and $L=2$
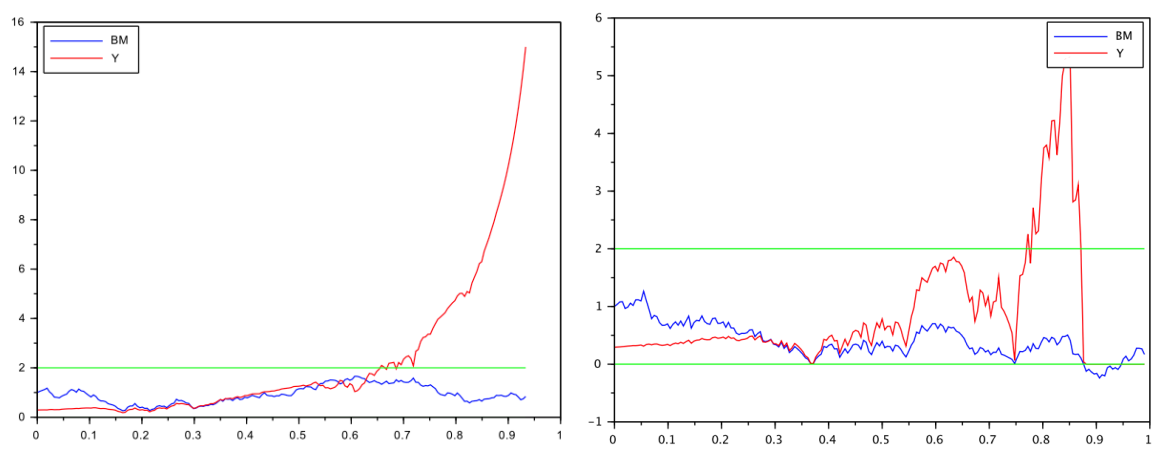

Figure 7: $\quad$ Two trajectories of $W$ and $Y$ (left with explosion, right without).

control problem to derive a sufficient (Lemma 7) and a necessary (Lemma 8) condition for the continuity $\lim _{t \rightarrow T} Y_{t}^{\min }=\xi$ of $Y^{\mathrm{min}}$ at $T$. Finally, we apply our findings from Sections 2 and 3 to derive estimates about the limiting behavior of conditional probabilities $\mathbb{P}_{t}[A]$ as $t \rightarrow T$ for $A=B(m, r)^{c} \in \mathscr{F}_{T}$ and $A=B(m, r) \in \mathscr{F}_{T}$ (Corollary 1).

Let us assume $p>1$ and as before $q$ denotes its Hölder conjugate; for an arbitrary $\xi \in \mathscr{F}_{T}, \xi \geq 0$, consider the stochastic optimal control problem

$$
\begin{aligned}
V(c, t, \omega) & \doteq \operatorname{essinf}_{\alpha \in \mathcal{A}(t, c)} \mathbb{E}\left[(p-1)^{p-1} \int_{t}^{T}\left|\alpha_{s}\right|^{p} d s+\left|C_{T}^{\alpha}\right|^{p} \xi \mid \mathscr{F}_{t}\right], \\
C_{u}^{\alpha} & \doteq c+\int_{t}^{u} \alpha_{s} d s, u \in[t, T],
\end{aligned}
$$

$t \in[0, T], c \in \mathbb{R}$, where the set of admissible controls $\mathcal{A}(t, c)$ consists of all progressively measurable processes $\alpha$ such that $\alpha \in L^{1}(t, T) \mathbb{P}$-a.s and we assume $0 \cdot \infty=0$. From the verification Theorems [1, Theorem 1.3] or [16, Theorem 3], we know that

$$
V(c, t, \omega)=|c|^{p} Y_{t}^{\min }
$$


where $Y^{\text {min }}$ is the minimal super-solution of the $\operatorname{BSDE}(3,4)$ constructed in these works ${ }^{4}$. In particular,

$$
\lim _{t \rightarrow T} V(c, t, \omega) \geq|c|^{p} \xi(\omega)
$$

holds $\mathbb{P}$-a.s. Moreover, an optimal control for (49) is given by $\alpha_{s}^{*}=-(q-1) C_{s}^{\alpha^{*}}\left|Y_{s}\right|^{q-1}$, and thus

$$
C_{u}^{\alpha^{*}}=c \exp \left[-(q-1) \int_{t}^{u}\left(Y_{s}\right)^{q-1} d s\right],
$$

for $t \leq u \leq T$. The link (50) between the value function $V$ and $Y^{\text {min }}$ will give two results concerning the continuity of $Y^{\mathrm{min}}$ in a general setting. First, we show $\left|\lim _{t \rightarrow T} Y_{t}^{\min }\right|<\infty$ already implies continuity.

Lemma 7. Let $Y^{\text {min }}$ be the minimal super-solution of the BSDE $(3,4)$. Then the following implication holds for almost all $\omega \in \Omega$. If

$$
\lim _{t \rightarrow T} Y_{t}^{\min }(\omega)<\infty
$$

then the path $Y^{\min }(\omega)$ is continuous at $T$, i.e. it holds that $\lim _{t \rightarrow T} Y_{t}^{\min }(\omega)=\xi(\omega)$.

Proof. Let $C_{t}=\exp \left(-(q-1) \int_{0}^{t}\left(Y_{s}^{\min }\right)^{q-1} d s\right)$ (which is the optimal control in (49) for $t=0$ and $c=1)$. Since $Y^{\mathrm{min}}$ is nonnegative, it follows that $C$ is continuous at $T: \lim _{t \rightarrow T} C_{t}=$ $C_{T} \in[0,1]$. We know from the analysis of the control problem (see [1], proof of Theorem 4.2) that

$$
Y_{t}^{\min } C_{t}^{p}=\mathbb{E}\left[(p-1)^{p-1} \int_{t}^{T}\left|\alpha_{s}\right|^{p} d s+\xi\left|C_{T}\right|^{p} \mid \mathscr{F}_{t}\right]
$$

with $\alpha_{s}=\frac{d C_{s}}{d s}=-(q-1)\left(Y_{s}^{\min }\right)^{q-1} C_{s}, s \in[t, T]$. The right side of (51) decomposes into a semimartingale $M+A$ with

$$
M_{t}=\mathbb{E}\left[(p-1)^{p-1} \int_{0}^{T}\left|\alpha_{s}\right|^{p} d s+\xi\left|C_{T}\right|^{p} \mid \mathscr{F}_{t}\right]
$$

and

$$
A_{t}=-(p-1)^{p-1} \int_{0}^{t}\left|\alpha_{s}\right|^{p} d s .
$$

Since $Y_{0}^{\min }=\mathbb{E}\left[M_{T}\right]<\infty$, the process $M$ is a true martingale. In particular, $M$ is continuous. $A$ is continuous by the fundamental theorem of calculus. Therefore, the right side of (51) converges to $M_{T}+A_{T}=\xi\left|C_{T}\right|^{p}$ as $t \nearrow T$, i.e.

$$
\lim _{t \nearrow T} Y_{t}^{\min } C_{t}^{p}=\xi\left|C_{T}\right|^{p} .
$$

Therefore, on the set $\left\{C_{T}>0\right\}$ we have $\lim _{t \nearrow_{T}} Y_{t}^{\min }=\xi$. Moreover, the definition of $C$ implies that if $C_{T}(\omega)=0$, then $\lim _{t \rightarrow T} Y_{t}^{\min }(\omega)=\infty$ and consequently $\left\{\lim _{t \rightarrow T} Y_{t}^{\min }<\right.$ $\infty\} \subseteq\left\{C_{T}>0\right\}$. This completes the proof.

Remark 2. The identity (51) is equivalent to the representation (29) in Lemma 4 (replace $Y_{t}^{\min }$ by $u\left(W_{t}, t\right)$ and take expectation).

\footnotetext{
${ }^{4}$ In [16] only the weak terminal constraint $\lim \inf _{t \rightarrow T} Y_{t}^{\text {min }} \geq \xi$ was established. In the present setting, it follows from the results in [23] that actually the $\operatorname{limit}_{\lim } \rightarrow T Y_{t}^{\mathrm{min}}$ exists and consequently $\lim _{t \rightarrow T} Y_{t}^{\mathrm{min}} \geq \xi$ holds.
} 
The next result gives a necessary condition for continuity. We use the shorthand notation $\mathbb{P}_{t}[\xi=\infty]=\mathbb{E}\left[\mathbf{1}_{\{\xi=\infty\}} \mid \mathscr{F}_{t}\right]$.

Lemma 8. Let $Y^{\mathrm{min}}$ be the minimal super-solution of the BSDE $(3,4)$ and suppose that continuity condition (8) holds for $Y^{\mathrm{min}}$. Then we have a.s. on $\{\xi<\infty\}$

$$
\sup _{t \in[0, T]} \frac{\mathbb{P}_{t}[\xi=\infty]}{(T-t)^{p-1}}<\infty
$$

Proof. For $t<T$ let $\alpha \in \mathcal{A}(t, 1)$ be an arbitrary strategy with associated position path $C^{\alpha}$ that has finite costs:

$$
\mathbb{E}\left[(p-1)^{p-1} \int_{t}^{T}\left|\alpha_{s}\right|^{p} d s+\left|C_{T}^{\alpha}\right|^{p} \xi \mid \mathscr{F}_{t}\right]<\infty
$$

Take for example the optimal strategy. Then we have

$$
\begin{aligned}
& \mathbb{E}\left[(p-1)^{p-1} \int_{t}^{T}\left|\alpha_{s}\right|^{p} d s+\left|C_{T}^{\alpha}\right|^{p} \xi \mid \mathscr{F}_{t}\right] \\
& \quad \geq(p-1)^{p-1} \mathbb{E}\left[\mathbf{1}_{\left\{C_{T}^{\alpha}=0\right\}} \int_{t}^{T}\left|\alpha_{s}\right|^{p} d s \mid \mathscr{F}_{t}\right] .
\end{aligned}
$$

Jensen's inequality yields $\int_{t}^{T}\left|\alpha_{s}\right|^{p} \geq \frac{1}{(T-t)^{p-1}}$ for every path satisfying $C_{T}^{\alpha}=0$. Moreover, since $\alpha$ has finite costs, it holds that $\{\xi=\infty\} \subseteq\left\{C_{T}^{\alpha}=0\right\}$. This implies

$$
\mathbb{E}\left[(p-1)^{p-1} \int_{t}^{T}\left|\alpha_{s}\right|^{p} d s+\left|C_{T}^{\alpha}\right|^{p} \xi \mid \mathscr{F}_{t}\right] \geq(p-1)^{p-1} \frac{\mathbb{P}_{t}[\xi=\infty]}{(T-t)^{p-1}} .
$$

Since the right side of the above display does not depend on the control $\alpha$, we use (50) to arrive at

$$
Y_{t}^{\min } \geq(p-1)^{p-1} \frac{\mathbb{P}_{t}[\xi=\infty]}{(T-t)^{p-1}} .
$$

Since $\lim _{t \rightarrow T} Y_{t}^{\min }<\infty$ if $\xi<\infty$, this yields the claim.

Lemma 8 combined with our results from Sections 2 and 3 allows to derive estimates on the speed of convergence $\lim _{t \rightarrow T} \mathbb{P}_{t}[A] \rightarrow 0$ on $A^{c}$ for $A=B(m, r)$ and $A=B(m, r)^{c}$. This is subject of the next corollary.

Corollary 1. Let $m \in \mathbb{R}$ and $r \in(0, \infty)$. Then on $B(m, r)$ it holds for all $p \in(1,2)$ that

$$
\sup _{t \in[0, T]} \frac{\mathbb{P}_{t}\left[B(m, r)^{c}\right]}{(T-t)^{p-1}}<\infty
$$

and on $B(m, r)^{c}$ it holds for all $p>1$ that

$$
\sup _{t \in[0, T]} \frac{\mathbb{P}_{t}[B(m, r)]}{(T-t)^{p-1}}<\infty .
$$

Proof. For the first result set $\xi=\infty \cdot \mathbf{1}_{B^{c}(m, r)}$ and let $\left(Y^{\min }, Z^{\min }\right)$ denote the minimal super-solution of $(3,4)$ and let $(Y, Z)$ denote the solution of $(3,4)$ constructed in Proposition 1. From Theorem $1, Y^{\mathrm{min}}=Y$ and in particular, it holds that $\lim _{t \rightarrow T} Y_{t}=\lim _{t \rightarrow T} Y_{t}^{\min }=\xi$. In other words continuity condition (8) holds. The first result then follows from Lemma 8 (observe that $q>2$ implies that $p<2$ ). The second result follows from the same argument and Lemma 8, this time used with the results in Section 3. 
This result is related to the notion of fractional smoothness developed by $[26,14]$, by [10] for applications in finance and by $[9,11]$ in the context of BSDEs. Indeed we get the following inequality: for $A=B^{c}(m, r)$ (and $p \in(1,2)$ ) or $A=B(m, r)$ (and $p>1$ ), it holds P-a.s.

$$
\forall t \in[0, T], \quad\left|\mathbf{1}_{A}-\mathbb{E}\left(\mathbf{1}_{A} \mid \mathcal{F}_{t}\right)\right| \leq C(T-t)^{p-1} .
$$

Following [9, Definition 1], this means that $\mathbf{1}_{A}$ belongs to $B_{q, \infty}^{2(p-1)}(W)$ for any $1<q<\infty$.

\section{Conclusion}

Let us comment on several direct extensions and possible future work. The extension of the boundary condition $\xi=\infty \cdot \mathbf{1}_{B^{c}}$ to $\infty \cdot \mathbf{1}_{B^{c}}+g\left(W_{T}\right) \mathbf{1}_{B}$ for $g$ such that $\mathbb{E}\left(\left|g\left(W_{T}\right)\right| \mathbf{1}_{B}\right)<\infty$ requires only that we change the terminal condition (13)

$$
V(0, t)=V(L, t)=y_{t}, t \in[0, T], V(x, T)=g(x), 0<x<L .
$$

Simple modifications of the argument of Section 2 would suffice to deal with this change. Generalizing the terminal condition $\xi=\infty \cdot \mathbf{1}_{B}$ to $\infty \cdot \mathbf{1}_{B}+g\left(W_{T}\right) \mathbf{1}_{B^{c}}$ for $g$ such that $\mathbb{E}\left(\left|g\left(W_{T}\right)\right| \mathbf{1}_{B}\right)<\infty$ requires the solution of two PDE: one must first solve (10) over the domain $\mathbb{R} \times[0, T]$ where $g$ serves as terminal condition on the terminal boundary of this domain. The value of the solution on $S=\{(L, t), t \in[0, T]\} \cup\{(0, t), t \in[0, T]\}$ will then serve as lateral boundary condition for the PDE (10) on $D$.

A further generalization involves changing the definition of the set $B$ to $\left\{\omega-r<X_{t}(\omega)-\right.$ $c<r, t \in[0, T]\}$ where $X$ is an SDE driven by $W$; this generalization would require to modify the second derivative term in (10) to the infitesimal generator of $X$. Further generalizations can consider the case when $X$ is an SDE with jumps or a doubly stochastic process, which may require further arguments and ideas. The treatment of these extensions may also be taken up in future work.

For the case when $\xi=\xi_{1}=\infty \cdot \mathbf{1}_{B^{c}}$ our arguments depended on $q>2$, which implied $\mathbb{E}\left[y_{\tau} \mathbf{1}_{\{\tau<T\}}\right]<\infty$. The work of Marcus \& Véron [20] and numerical computations suggest that even when $q \in(1,2]$ the PDE (10) and the boundary condition (13) have a smooth solution. Future work can also try to treat the terminal condition $\xi_{1}$ with $q \in(1,2]$.

The present work doesn't address the question of uniqueness of the solution of the BSDE $(3,4)$ for the terminal condition $\xi=\xi_{2}=\infty \cdot \mathbf{1}_{B}$; even the uniqueness of the solution of the associated PDE (10) and the boundary condition (44) appears to be open. The most relevant works on this question are $[19,20]$ and, to the best of our understanding, the results in these works do not settle the question of uniqueness for the PDE. Therefore, the question of uniqueness for the terminal condition $\xi=\xi_{2}$ is for the moment open and can be a direction for future research.

The single space dimension that we have treated in the present work simplified our existence and smoothness arguments for the solutions of the PDE we have studied. Their extension to higher dimensions could also be the subject of future work. In this, a possible approach is, as hinted at in the introduction, to develop arguments for our PDE problems starting from results of [19].

Finally, from an applied perspective, we think that it would be of interest to study the implications of the results of the current work for the portfolio liquidation problem mentioned in Section 4. 


\section{References}

[1] S. Ankirchner, M. Jeanblanc, and T. Kruse, BSDEs with singular terminal condition and a control problem with constraints, SIAM Journal on Control and Optimization 52 (2014), no. 2, 893-913.

[2] P. Baras and M. Pierre, Problèmes paraboliques semi-linéaires avec données mesures, Applicable Anal. 18 (1984), no. 1-2, 111-149.

[3] H. Brézis and A. Friedman, Nonlinear parabolic equations involving measures as initial conditions, J. Math. Pures Appl. (9) 62 (1983), no. 1, 73-97.

[4] Ph. Briand, B. Delyon, Y. Hu, E. Pardoux, and L. Stoica, $L^{p}$ solutions of backward stochastic differential equations, Stochastic Process. Appl. 108 (2003), no. 1, 109-129.

[5] R. Douady, Closed form formulas for exotic options and their lifetime distribution, International Journal of Theoretical and Applied Finance 2 (1999), no. 01, 17-42.

[6] E. B. Dynkin and S. E. Kuznetsov, Trace on the boundary for solutions of nonlinear differential equations, Trans. Amer. Math. Soc. 350 (1998), no. 11, 4499-4519.

[7] L. C. Evans, Partial differential equations, second ed., Graduate Studies in Mathematics, vol. 19, American Mathematical Society, Providence, RI, 2010.

[8] A. Friedman, Partial differential equations of parabolic type, Dover Publications, 2008.

[9] C. Geiss, S. Geiss, and E. Gobet, Generalized fractional smoothness and $L_{p}$-variation of BSDEs with non-Lipschitz terminal condition, Stochastic Process. Appl. 122 (2012), no. 5, 2078-2116. MR 2921973

[10] S. Geiss and E. Gobet, Fractional smoothness and applications in finance, Advanced mathematical methods for finance, Springer, Heidelberg, 2011, pp. 313-331. MR 2792085

[11] _ Fractional smoothness of functionals of diffusion processes under a change of measure, Electron. Commun. Probab. 19 (2014), no. 35, 14. MR 3225866

[12] P. Graewe, U. Horst, and J. Qiu, A non-markovian liquidation problem and backward spdes with singular terminal conditions, SIAM Journal on Control and Optimization 53 (2015), no. 2, 690-711.

[13] P. Graewe, U. Horst, and E. Séré, Smooth solutions to portfolio liquidation problems under price-sensitive market impact, Stochastic Processes and their Applications (2017).

[14] F. Hirsch, Lipschitz functions and fractional Sobolev spaces, Potential Anal. 11 (1999), no. 4, 415-429. MR 1719833

[15] I. Karatzas and S. E. Shreve, Brownian motion and stochastic calculus, second ed., Graduate Texts in Mathematics, vol. 113, Springer-Verlag, New York, 1991.

[16] T. Kruse and A. Popier, Minimal supersolutions for BSDEs with singular terminal condition and application to optimal position targeting, Stochastic Processes and their Applications 126 (2016), no. 9, 2554 - 2592. 
[17] O. A. Ladyženskaja, V. A. Solonnikov, and N. N. Ural'ceva, Linear and quasilinear equations of parabolic type, Translated from the Russian by S. Smith. Translations of Mathematical Monographs, Vol. 23, American Mathematical Society, Providence, R.I., 1968.

[18] J.-F. Le Gall, A probabilistic approach to the trace at the boundary for solutions of a semilinear parabolic partial differential equation, J. Appl. Math. Stochastic Anal. 9 (1996), no. 4, 399-414.

[19] M. Marcus and L. Véron, Initial trace of positive solutions of some nonlinear parabolic equations, Communications in Partial Differential Equations 24 (1999), no. 7-8, 14451499.

[20] M. Marcus and L. Véron, Semilinear parabolic equations with measure boundary data and isolated singularities, J. Anal. Math. 85 (2001), 245-290.

[21] A. Matoussi, L. Piozin, and A. Popier, Stochastic partial differential equations with singular terminal condition, Stochastic Process. Appl. 127 (2017), no. 3, 831-876. MR 3605713

[22] E. Pardoux and S. Peng, Backward stochastic differential equations and quasilinear parabolic partial differential equations, Stochastic partial differential equations and their applications, Springer, 1992, pp. 200-217.

[23] A. Popier, Backward stochastic differential equations with singular terminal condition, Stochastic Processes and their Applications 116 (2006), no. 12, 2014-2056.

[24] L Limit behaviour of BSDE with jumps and with singular terminal condition, ESAIM Probab. Stat. 20 (2016), 480-509. MR 3581831

[25] _ Integro-partial differential equations with singular terminal condition, Nonlinear Anal. 155 (2017), 72-96. MR 3631743

[26] S. Watanabe, Fractional order Sobolev spaces on Wiener space, Probab. Theory Related Fields 95 (1993), no. 2, 175-198. MR 1214086 